\title{
Summary and Outlook: 2015 Lepton-Photon Symposium
}

\section{John Ellis*广}

Theoretical Particle Physics and Cosmology Group, Department of Physics, King's College London, Strand, London WC2R 2LS, U.K;

Theory Division, Physics Department, CERN, CH 1211 Geneva 23, Switzerland

E-mail: John.Ellis@cern.ch

What life is there after Higgs? Specifically, what physics lies beyond the Standard Model (SM)? These are the biggest questions in particle physics today, and my talk is oriented towards efforts to answer them at the LHC and elsewhere.

XXVII International Symposium on Lepton Photon Interactions at High Energies 17-22 August 2015

Ljubljana, Slovenia

\footnotetext{
* Speaker.

${ }^{\dagger}$ Work supported in part by the European Research Council via the Advanced Investigator Grant 267352 and by the UK STFC via the research grant ST/L000326/1.
} 


\section{Introduction}

Fig. 1 outlines the plan of this talk. There are many reasons to think that there must be physics beyond the SM, including the apparent instability of the electroweak vacuum, the existence of dark matter, the origin of the cosmological matter-antimatter asymmetry, the masses of neutrinos, the hierarchy of mass scales in physics, cosmological inflation and the need for a quantum theory of gravity. The long-term future of particle physics hinges upon the nature of whatever physics lies beyond the SM, and how we can discover and study it. As discussed in successive Sections of this talk, our quest for this new physics must start from our knowledge of the SM and be advised by theoretical ideas what may lie beyond it. As well as the LHC, our tools for discovering and studying it include high-intensity low-energy experiments as well as astroparticle physics and future experiments at the high-energy frontier.

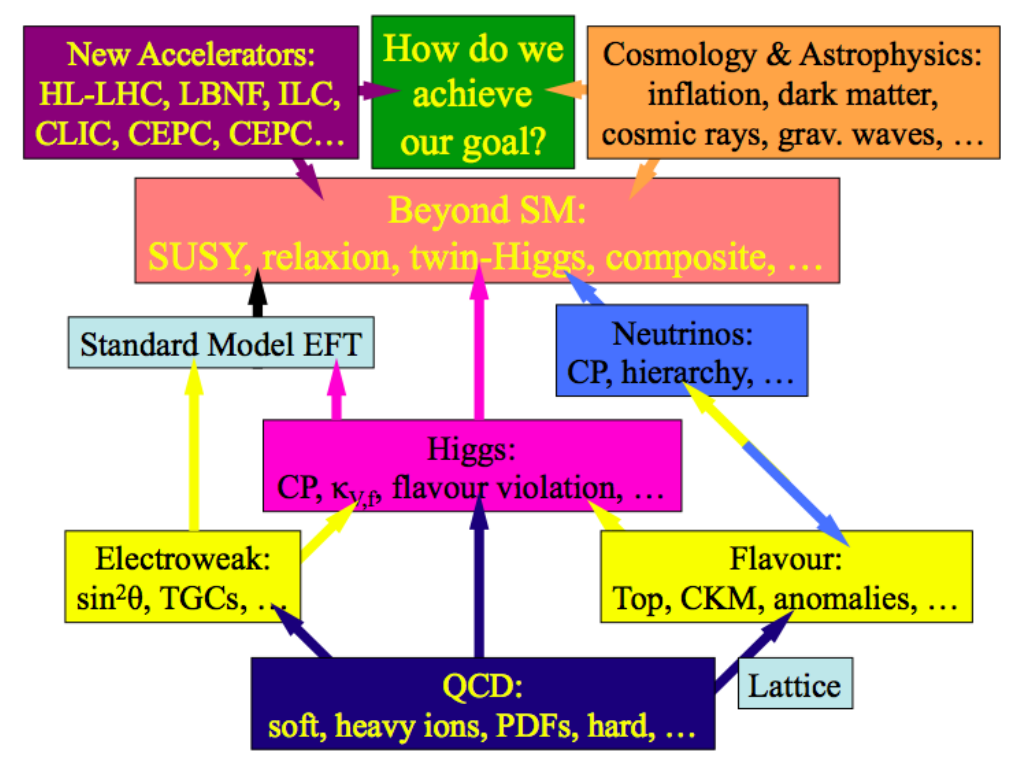

Figure 1: Our search for physics beyond the $S M$ is rooted in our understanding of the $S M$, and will require input from astroparticle and accelerator experiments, as well as define their future agendas.

\section{QCD}

Understanding QCD is the essential basis for physics at the LHC, including the search for any physics beyond the SM. QCD provides backgrounds, in the forms of the underlying events, jets and pile-up, it provides tests of the SM via multi-jet, $W$, and $Z$ production, it is crucial for unravelling top physics, notably for determining the top mass, and precision QCD calculations of hard processes are essential for calculating the production of new particles such as the Higgs boson, distinguishing them from backgrounds and studying them quantitatively.

However, most QCD processes at the LHC are soft: they cannot, in general, be calculated accurately from first principles, and cosmic-ray studies as well as QCD physics need them to be modelled accurately [1]. Run I of the LHC has already helped cosmic-ray physicists [2] by measuring and reducing the extrapolation uncertainties in the total and inelastic $p p$ cross-sections. 
The improved modelling of soft QCD has reduced the uncertainty in the nuclear composition of ultra-high-energy cosmic rays, and additional help could be provided by measuring $p$-Oxygen collisions at the LHC. The first LHC Run-II paper contained measurements of the multiplicity and pseudo-rapidity distribution at $13 \mathrm{TeV}[3,4]$, which provide further discrimination between models: currently, EPOS-LHC seems to provide the best available description.

Parton distribution functions (PDFs) provide the bridge between soft and hard QCD, and this Symposium witnessed significant reductions in their spread and uncertainties [5, 6]. In particular, the uncertainty in the gluon-gluon luminosity function relevant for the dominant Higgs production mechanism is now $\mathscr{O}(2) \%$, thanks in particular to input from HERA, as seen in the left panel of Fig. 2. This uncertainty is matched by the uncertainty in the $g g \rightarrow H$ subprocess cross-section, which has now been calculated to $\mathrm{N}^{3} \mathrm{LO}$ and has an uncertainty $\mathscr{O}(3) \%$ [8]. The resultant improvement in the Higgs production cross-section will enable a new era in precision Higgs physics, improving the sensitivity to new physics beyond the SM. Many other processes, including kinematic distributions for Higgs production, have been calculated to NNLO, and some NLO electroweak calculations are also available [6, 7], making possible other measurements of Higgs couplings.
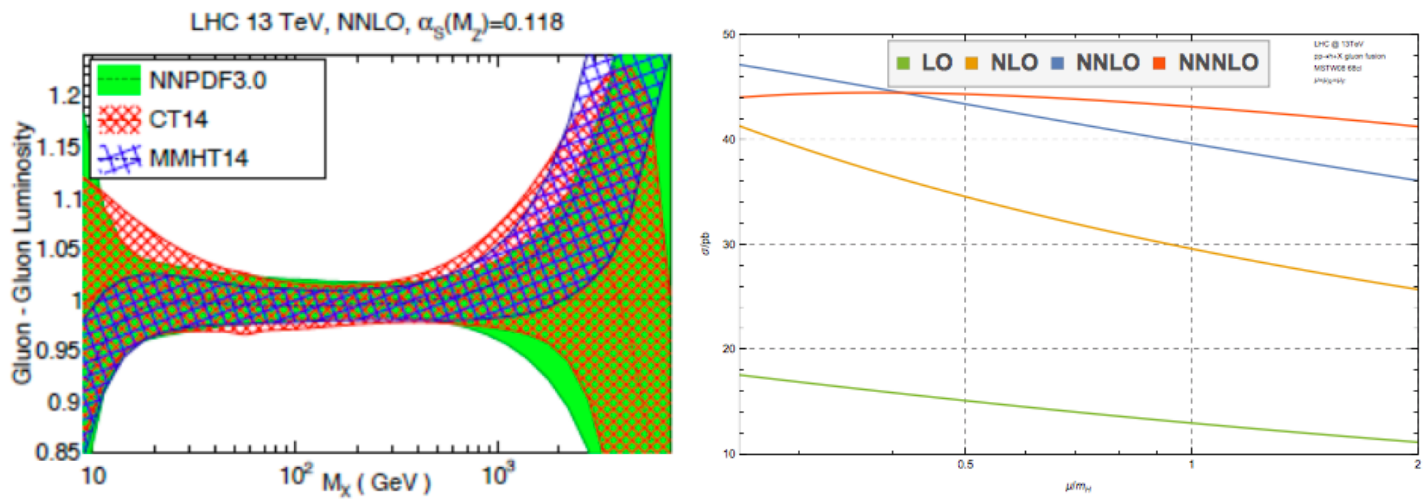

Figure 2: Left panel: Recent determinations of the gluon-gluon luminosity function for Higgs production at $13 \mathrm{TeV}$ agree to within $\mathscr{O}(2) \%$ [5, 6]. Right panel: The renormalization-scale dependence of the NNNLO cross-section for $g g \rightarrow H$ leads to an estimated theoretical uncertainty in is $\mathscr{O}(3) \%$ [8].

Perturbative QCD calculations of jet cross-sections agree with the data over many orders of magnitude, and make possible an interesting measurement of $\alpha_{s}$ that is consistent with the world average. Moreover, they show that $\alpha_{s}$ runs beyond the TeV scale: into a GUT? Many other crosssections for the production of $W, Z$ bosons and $\bar{t} t$ pairs with varying numbers of jets, as well as subdominant Higgs production processes, also show excellent agreement between theory and experiment.

One of the key issues in QCD is the determination of the top mass, which is fundamental in itself but also vital for our understanding of the stability of the electroweak vacuum, as discussed later. The LHC and Tevatron experiments have announced a world average value [9]:

$$
m_{t}=173.34 \pm 0.76 \mathrm{GeV},
$$

representing a determination at the $\mathscr{O}(1 / 2) \%$ level. But what have they determined, and how is it related to the short-distance mass in the SM Lagrangian? The good news is that the relationship 
between the pole definition of $m_{t}$ and the running mass is well under control at NNNNLO [10, 11]:

$$
\begin{aligned}
m_{t}^{\text {rmpole }} & =m_{t}^{\text {running }}\left(1+0.4244 \alpha_{s}+0.8345 \alpha_{s}^{2}+2.375 \alpha_{s}^{3}+(8.49 \pm 0.25) \alpha_{s}^{4}+\ldots\right), \\
& =m_{t}^{\text {running }}+(7.557+1.617+0.501+(0.195 \pm 0.005)+\ldots) \mathrm{GeV}
\end{aligned}
$$

where the $(\ldots)$ represent uncalculated higher-order terms. However, the relationship between the pole mass and the definition of $m_{t}$ used in Monte Carlo codes is less clear: non-perturbative effects may be important, though no obvious bias has been seen so far. At the moment, the experimental uncertainty in (2.1) may be dominant, but new measurements have been reported [12]:

$$
\begin{aligned}
m_{t}= & 174.98 \pm 0.58 \pm 0.49 \mathrm{GeV} & {[D 0], } \\
& 172.99 \pm 0.91 \mathrm{GeV} & {[\text { ATLAS }], } \\
& 172.44 \pm 0.49 \mathrm{GeV} & {[C M S[13]], }
\end{aligned}
$$

and the overall error in $m_{t}$ in (2.1) may be reduced by $\mathscr{O}(2)$ during LHC Run II, in which case better understanding will be needed.

To pentaquark or not to pentaquark? That is the question raised by the discovery of two states with $\bar{c} c u u d$ quantum numbers reported recently by LHCb [14], one of which has a very nice resonance-like Argand diagram, as seen in Fig. 3. But are they true pentaquark states in a single 'bag', or are they loosely-bound 'di-bag' 'molecular' states? The latter interpretation may be supported by fact that the $4450 \mathrm{MeV}$ state has a small decay width, despite having $\mathscr{O}(400) \mathrm{MeV}$ of phase space to decay into, and the fact that its mass is barely below $m_{\bar{\Sigma}_{c}}+m_{D^{*}}[16]$, just as there are several 'tetraquark' states close to charm meson-antimeson thresholds [15]. If it is a 'molecule', many more 'pentaquarks' may be around the corner, and their structure may soon be elucidated. Estimates suggest that they could be produced plentifully and observable in photoproduction experiments at JLAB, in particular [17].
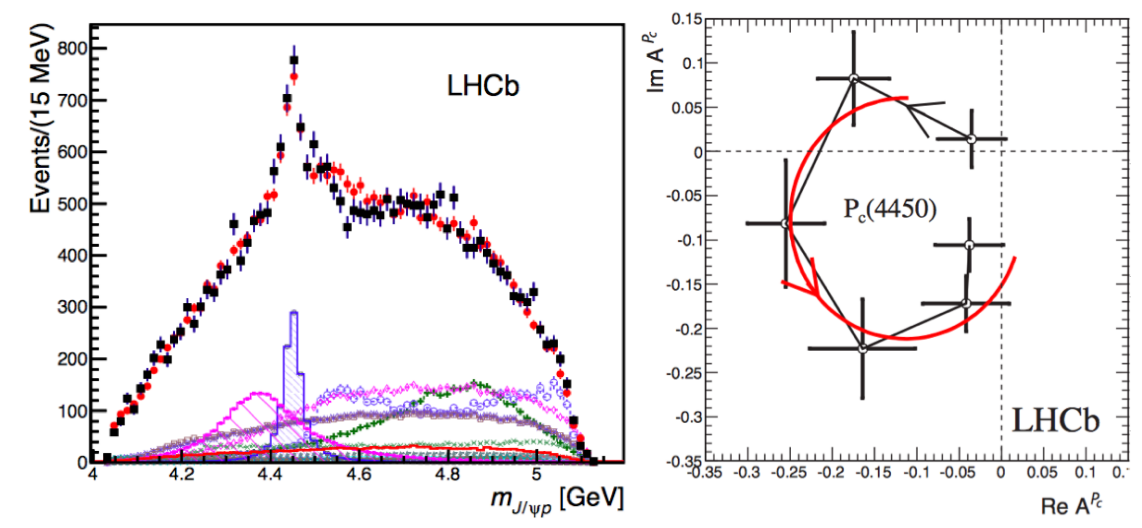

Figure 3: Left panel: Fitting the $L H C b m_{J / p s i p}$ mass distribution requires two exotic states at $4380 \mathrm{MeV}$ and $4450 \mathrm{MeV}$ [14]. Right panel: The Argand diagram for the $4450 \mathrm{MeV}$ state exhibits the behaviour expected for a Breit-Wigner resonance [14].

Relativistic heavy-ion collisions are not directly relevant to searches for new physics beyond the SM, but they may provide an arena for probing predictions obtained using the AdS/CFT correspondence originating from string theory [18]. Lattice calculations indicate that there is no phase 
transition between hadronic and quark-gluon matter, but a crossover at $T_{c}=155 \pm 5 \mathrm{MeV}$ [19], which is consistent with measurements at RHIC and LHC [20]. It is expected that $T_{c}$ should decrease as the baryon chemical potential $\mu_{B}$ increases, and that a phase boundary should appear at a critical end-point at some $\mu_{B} \neq 0$. Its location cannot yet be calculated reliably, but many experiments (RHIC, CERN SPS, NICA, FAIR) are underway or planned to search for it. Indications are that the medium beyond the crossover is perhaps the most perfect fluid known [18]. Evidence for this is provided by measurements of azimuthal anisotropies $v_{n}$ that can be explained by hydrodynamic flow with a very low viscosity-to entropy ratio $\eta / s<0.2$. This very close to the lower bound $\eta / s=1 / 4 \pi$ from the stringy AdS/CFT correspondence [21], which may be useful for calculating other properties of this fluid.

This stringy hydrodynamic picture works very well for azimuthal anisotropies in relativistic ion-ion collisions, and an analogous (?) collective near-side 'ridge' is also seen in $p$-Lead collisions. But it came as a surprise when CMS observed an analogous near-side ridge in highmultiplicity $p p$ collisions at $8 \mathrm{TeV}$ [22], which has now been seen also by ATLAS at $13 \mathrm{TeV}$ [23, 25]. Does this mean that hydrodynamics applies also to $p p$ collisions? If so, are AdS/CFT ideas applicable? Is it evidence for a colour-glass condensate [24]? It is to be hoped that the detailed studies possible during LHC Run II will provide answers.

Many other collective effects have been seen in relativistic heavy-ion collisions, including the suppression of particle production, jet quenching (which is not seen in $p$-Lead collisions), $\Upsilon$ suppression, as well as $J / \Psi$ suppression at RHIC and regeneration at the LHC [20]. They exhibit a rich landscape that we are only beginning to explore [18].

\section{Flavour Physics}

Flavour measurements are generally in good agreement with the Cabibbo-Kobayashi-Maskawa (CKM) description in the SM [26, 27], and the unitarity triangle continues to be alive and well in 2015 (with crucial input from many lattice calculations [29]), as seen in the left panel of Fig. 4. The CKM picture has made many successful predictions, e.g., for many modes of CP violation in $B$ meson decays, and also predicted successfully (unfortunately) that $\mathrm{CP}$ violation in the charm sector would be below the present experimental sensitivity. However, the data still allow substantial contributions to $B$ physics from effects beyond the SM [27], and its still seems to me an open question whether all $\mathrm{TeV}$ physics must copy CKM slavishly as in models with minimal flavour violation.

Consider for example $B_{s, d} \rightarrow \mu^{+} \mu^{-}$decay [30]. The discovery of $B_{s} \rightarrow \mu^{+} \mu^{-}$decay by $\mathrm{LHCb}$ and CMS at the predicted level was a tremendous success for CKM [31]. However, these experiments also provide a hint that $B_{d} \rightarrow \mu^{+} \mu^{-}$decay may occur at a level higher than predicted by CKM, which would require non-minimal flavour violation.

There are several other anomalies in flavour physics, summarized in the right panel of Fig. 4, which one may or may not take seriously. One of the most intriguing is a hint for $H \rightarrow \tau \mu$ decay [32], of which more later. Another is an apparent violation of $e \mu$ universality in $B \rightarrow K \ell^{+} \ell^{-}$ decay [27]: this would be such a surprise that my attitude is 'wait and see', and the same remark applies to the reported evidence for a violation of lepton universality in $B \rightarrow D^{(*)} \tau \nu$ decay. Concerning the apparent anomaly in the $P_{5}^{\prime}$ angular distribution in $B \rightarrow K^{*} \mu^{+} \mu^{-}$decay [27], recent theoretical calculations [33] indicate that the QCD uncertainties may have been underestimated 

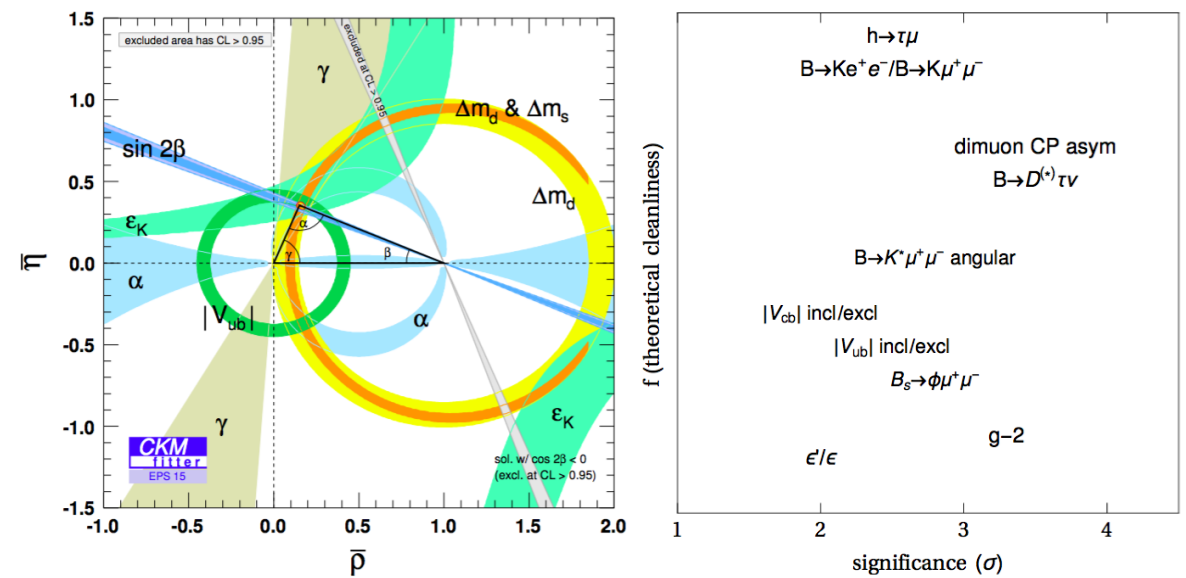

Figure 4: Flavour physics data are generally consistent with Cabibbo-Kobayashi-Maskawa predictions (left panel), but there are a number of apparent anomalies (right panel) [27, 26].

previously [34]. For the same reason, I propose to 'wait and see' whether the apparent excess in the $q^{2}$ distribution for $B \rightarrow \phi \mu^{+} \mu^{-}$decay will survive. I also note that updated measurements of the dimuon $A_{F B}$ asymmetry measured at the Tevatron no longer seem to be in serious conflict with SM expectations [28], whereas the CP asymmetry remains an open issue I would take a relaxed attitude towards the possibility of a violation of CKM unitarity until the discrepancies between the inclusive and exclusive determinations of $\left|V_{u b, c b}\right|$ [29] are ironed out, and I am not losing any sleep over $\varepsilon^{\prime} / \varepsilon$, which is notoriously tricky to calculate accurately [35].

In addition to $B$ physics, there are other interesting experimental probes of flavour physics. These include measurements of $K \rightarrow \pi \bar{v} v$ decay in both the charged and neutral channels [36]. The SM makes quite specific predictions for the branching ratios for these decays, which can be explored in forthcoming experiments. There is currently significant scope for new physics beyond the SM, particularly in the neutral mode.

Another exciting area is that of the electric dipole moments (EDMs), which violate CP. There has recently been significant progress by the ACME group in constraining the electron EDM: $d_{e}<8.7 \times 10^{-29}$ e-cm [37], which is an important constraint on many new physics models [36]. However, its significance for supersymmetry should not be overstated: the CP-violating phases that enter one-loop supersymmetric diagrams contributing to EDMs have completely different origins from the Kobayashi-Maskawa phase, and there are possibilities of cancellations between the phases contributing to the EDMs that are constrained by current experiments, which could leave open measurable possibilities for other quantities [38].

An open possibility is that of charged-lepton-flavour violation (CLFV) [39]. This is to be expected given the observation of neutrino oscillations, but would be unobservably small in the SM. However, it could be observable in some extensions of the SM, such as supersymmetric models with neutrino masses, so it is good news that MEG plans an upgrade of its search for $\mu \rightarrow e \gamma$, and that renewed searches for $\mu \rightarrow e e e$ and $\mu N \rightarrow e N$ are also planned. In parallel, flavour factories offer opportunities to improve the sensitivities to CLFV in $\tau$ decays.

Before leaving this Section, I would like to mention two other searches for new physics in lowenergy physics: $g_{\mu}-2$ and the charge radius of the proton [36]. Over a decade after the emergence 
of a substantial discrepancy between the experimental measurement of $g_{\mu}-2$ and SM calculations based on low-energy $e^{+} e^{-}$annihilation and $\tau$ decay data, we are no closer to a resolution. The good news is a new $g_{\mu}-2$ experiment is taking shape at Fermilab [40], that there are also possibilities at JPARC, and that new $e^{+} e^{-}$data offer the prospect of substantial reduction in the uncertainty in the SM calculation. As discussed later, if it holds up the $g_{\mu}-2$ discrepancy could be a harbinger of new physics at the TeV scale, such as supersymmetry. On the other hand, it is difficult for me to imagine a plausible new physics scenario for the anomaly in the proton charge radius [41].

\section{Electroweak Physics at the Tevatron and LHC}

The 'gold standard' for electroweak measurements has been provided for over a decade by LEP and the SLC [42]. However, the forward-backward asymmetry in $Z$ production at the Tevatron already gives precision in the electroweak mixing angle $\sin ^{2} \theta$ that is in the same ball-park as individual LEP and SLC measurements, and the Tevatron experiments could do better by combining all the CDF and D0 results using both $e^{+} e^{-}$and $\mu^{+} \mu^{-}$measurements [43]. The LHC experiments ATLAS, CMS and now LHCb [44] have entered the precision electroweak game, but still have much larger uncertainties, and it will be a long-term challenge to make a competitive determination of $\sin ^{2} \theta$.

Another class of electroweak measurements is provided by (anomalous) triple gauge couplings (TGCs), where LEP has led the way so far. LHC measurements of $W^{+} W^{-}, W \gamma$ and $W Z$ final states in Run I at $8 \mathrm{TeV}$ are already providing competitive constraints on $\Delta \kappa_{\gamma}, \lambda_{\gamma}, \Delta \kappa_{Z}, \lambda_{Z}$ and $\Delta g_{1}^{Z}$, LHC measurements of $Z \gamma$ and $Z Z$ final states constrain other TGCs significantly better than previous measurements and is starting to constrain quartic gauge couplings [43], and Run II will provide significant improvements in the sensitivities to all TGCs. In addition to their intrinsic importance, the TGC constraints are important ingredients, together with precision electroweak data and Higgs measurements, in constraining effective field theories (EFTs) of possible physics beyond the SM, as discussed later.

\section{Higgs Measurements}

The Higgs boson may be the most direct portal to physics beyond the SM.

The most accurate measurements of the Higgs mass are in the $Z Z^{*} \rightarrow 4 \ell^{ \pm}$and $\gamma \gamma$ final states. ATLAS and CMS have now combined their results $[45,46]$, as seen in the left panel of Fig. 5 to yield a combined value

$$
m_{H}=125.09 \pm 0.21 \text { stat. } \pm 0.11 \text { syst. . }
$$

It is noteworthy that statistical uncertainties still dominate, and one can expect that Run II measurements will bring the overall uncertainty down to $\mathscr{O}(100) \mathrm{MeV}$. In addition to its intrinsic importance, accuracy in $m_{H}$ enables precision measurements of couplings, since some Higgs decay rates vary rapidly as functions of $m_{H}$. Also, like $m_{t}$, reducing the uncertainty in $m_{H}$ is crucial for assessing the stability of the electroweak vacuum within the SM, as discussed later.

The ATLAS and CMS Collaborations have now also released a combination of their results on Higgs couplings [47]. In particular, they have checked the extent to which the Higgs couplings to massive particles are proportional to their masses, via parameterizations of the forms $\kappa_{F} m f / v$ 

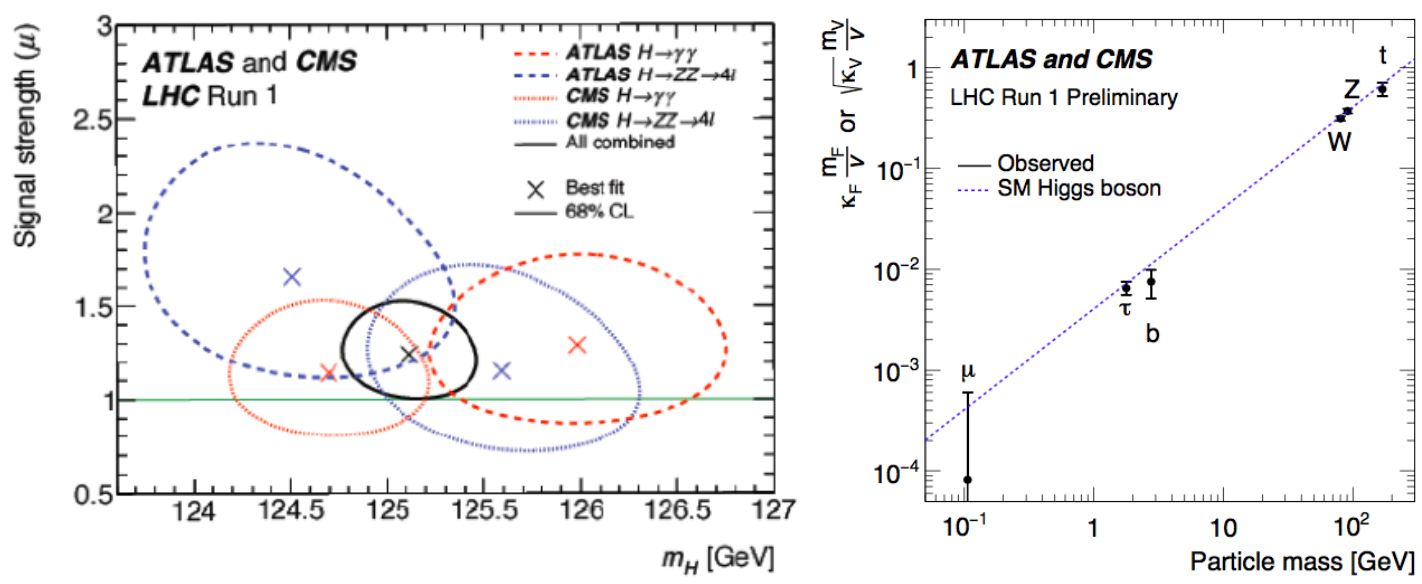

Figure 5: Left panel: The combination of the ATLAS and CMS measurements of $m_{H}$ [45, 46]. Right panel: Test by ATLAS and CMS of the expected linear dependence of the Higgs couplings to other particles on their masses [47].

for fermions and $\sqrt{\kappa_{V}} m_{V} / v$ for the $W^{ \pm}$and $Z^{0}$, where $\kappa_{F}=\kappa_{V}=1$ in the SM. As seen in the right panel of Fig. 5, their combined data are currently consistent with the expected linear mass dependence, with the normalization $v=246 \mathrm{GeV}$ expected in the SM.

Both ATLAS and CMS have also made extensive checks of the spin-parity of the Higgs boson using $Z Z^{*}, W W^{*}$ and $\gamma \gamma$ final states [46]. Alternatives to the expected $0^{+}$spin-parity assignment are excluded at $>99.9 \% \mathrm{CL}$. Combined CDF and D0 data from the Tevatron also exclude pure $0^{-}$ and $2^{+}$spin-parity assignments, if the same signal strength as in the SM is assumed [48].

The signal strengths $\mu_{i}$ relative to the SM predictions measured by ATLAS and CMS in various channels are shown in the left panel of Fig. 6 [46], with the overall results

$$
\begin{aligned}
& \mu=1.18_{-0.14}^{+0.15} \quad[\text { ATLAS }], \\
& 1.00 \pm 0.14 \quad[C M S], \\
& 1.09_{-0.10}^{+0.11} \quad \text { [Combined [47]], }
\end{aligned}
$$

which are clearly completely compatible with the SM at the $10 \%$ level. The dominant sources of uncertainties in the combination are experimental statistics and the theoretical uncertainty in the signal calculations. Run II will provide much increased statistics and a substantial reduction in the signal strength uncertainty is already in hand, so a reduction in the combined error by a factor $\sim 2$ seems attainable with Run II.

A fly in this SM Higgs ointment may be provided by flavour-changing Higgs couplings. These would be very small in the SM or its minimal supersymmetric extension (the MSSM) but all one can say model-independently is that Higgs exchange should not violate upper bounds on lowenergy flavour-changing interactions. These exclude observable quark-flavour-violating $H$ decays, but lepton-flavour-violating $H$ decays could be large: $H \rightarrow$ either $\mu \tau$ or $e \tau$ could have a branching ratio $\mathscr{B}=\mathscr{O}(10) \%$, though $\mathscr{B}(H \rightarrow e \mu)$ must be $<2 \times 10^{-5}[49,50]$. First CMS (see the right panel of Fig. 6 [32]) and now ATLAS [51] have reported a constraint on $\mathscr{B}(H \rightarrow \mu \tau)$ :

$$
\mathscr{B}(H \rightarrow \mu \tau)=0.84_{-0.37}^{+0.39} \% \quad[C M S],
$$



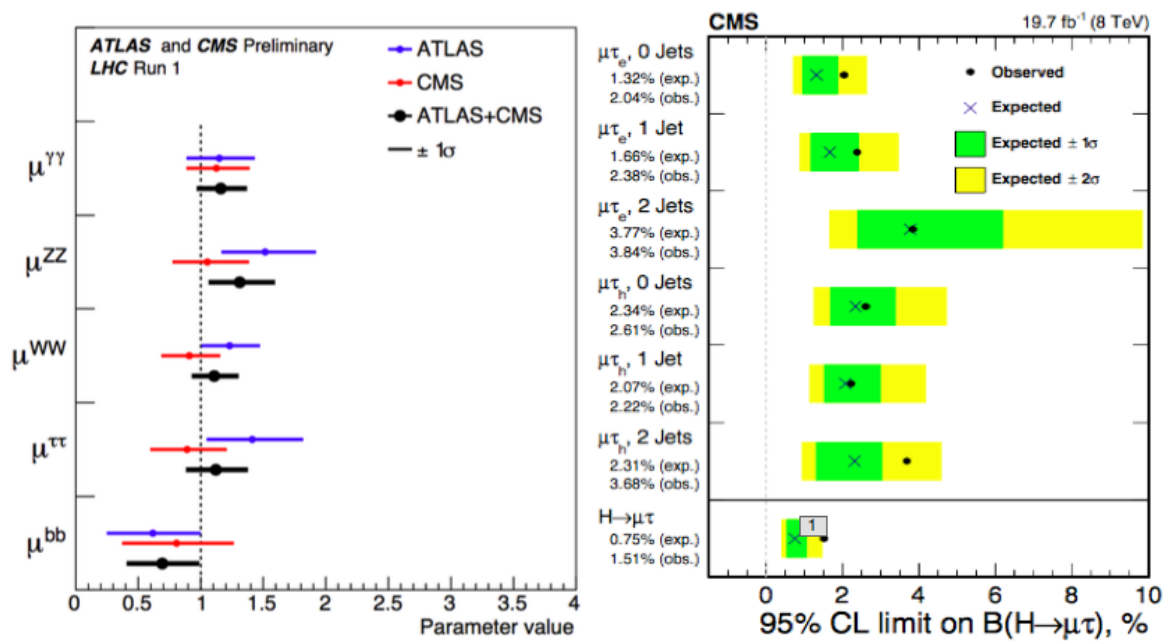

Figure 6: Left panel: The combination of the ATLAS and CMS measurements of the Higgs signal strengths $\mu$ [46, 47]. Right panel: Measurement by CMS of the $H \rightarrow \mu \tau$ branching ratio [32].

$$
0.77 \pm 0.63 \% \quad[A T L A S]
$$

It would be premature to take seriously this hint at the level of $\sim 2.5 \sigma$, which could only be accommodated by dint of a surprising complication of the SM Higgs sector, but let us wait and see. Meanwhile, CMS has also established upper limits $\mathscr{B}(H \rightarrow e \tau) \%$ and $\mathscr{B}(H \rightarrow e \mu)<0.036 \%$ [52], which do not challenge our prejudices [49, 50].

What do these measurements tell us about the nature of the Higgs boson, and specifically whether it is elementary or composite? Generally speaking, there have been two main schools of thought. If it is elementary, the systematic way to tame quadratic divergences in loop corrections to its mass is to postulate supersymmetry, in which case the residual corrections to its couplings are expected to be perturbatively small. Alternatively, in a composite Higgs scenario based on some new strong interactions, one might expect the corrections to the SM predictions for Higgs couplings to be larger, even if one postulates some approximate higher symmetry with the Higgs interpreted as a pseudo-Nambu-Goldstone boson (PNGB).

One convenient way to analyze this and other models containing a Higgs-like particle is to introduce factors $\kappa_{V, F}$ modifying its couplings to bosons and fermions, normalized to unity in the SM [53]. Whereas some production and decay modes are insensitive to the signs of $\kappa_{V, F}$, e.g., $H W$ associated production or $H \rightarrow \tau^{+} \tau^{-}$, others are sensitive to their relative signs, e.g., $H \rightarrow \gamma \gamma$ or $t H$ associated production. As could be expected from the results (5.4), the combined global analysis by ATLAS and CMS is consistent with the SM prediction $\left(\kappa_{V}, \kappa_{F}\right)=(1,1)$, as seen in Fig. 7, and the negative relative sign is excluded by almost $5 \sigma$ [47]. Moreover, there is good consistency between these direct constraints on the Higgs couplings and indirect constraints coming from precision electroweak data [7, 54]. They imply that the parameters of simple PNGB models must be tuned to resemble the SM at the $\mathscr{O}(10) \%$ level [46], as well also as avoiding the prediction of heavier Higgs-like bosons [55].

An alternative approach to searching for possible deviations from the SM is to assume the SM couplings of all particles including the Higgs boson at the level of the renormalizable terms in the 

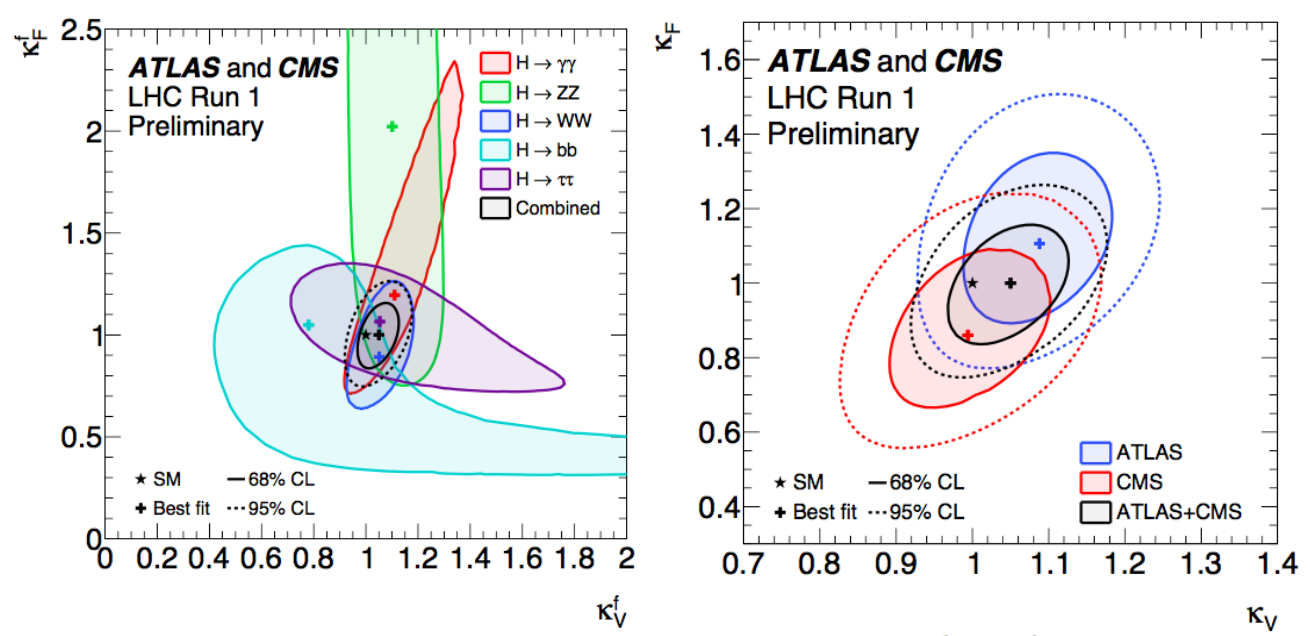

Figure 7: Left panel: The combination of the ATLAS and CMS measurements of $\left(\kappa_{V}, \kappa_{F}\right)$ in each measured channel [47]. Right panel: The combination of the ATLAS and CMS global fits to $\left(\kappa_{V}, \kappa_{F}\right)$ [47].

Lagrangian, but allow for for higher-dimensional operators combining these fields as might appear as relics of higher-energy physics, the SM effective field theory (EFT) approach [7, 54]:

$$
\mathscr{L}=\mathscr{L}_{\mathrm{SM}}+\mathscr{L}_{\text {eff }}: \quad \mathscr{L}_{\text {eff }}=\sum_{n} \frac{f_{n}}{\Lambda^{2}} \mathscr{O}_{n}^{(6)}+\ldots
$$

where the $\mathscr{O}_{n}^{(6)}$ are a complete set of dimension-6 operators, and the ... represent terms of dimension 8 and beyond. There are many such operators, 2499 in the general case, reducing to 59 if one assumes flavour conservation and family universality, of which 10 are purely bosonic, but there are also many ways to constrain their coefficients, including precision electroweak data, TGCs and kinematic distributions in Higgs production and decay, as well as the magnitudes of Higgs couplings.

One example of an analysis where the effects of dimension- 6 operators may be important is in the interpretation of off-shell Higgs production and the constraint it imposes on the total Higgs width, $\Gamma_{H}$ [7]. If the off-shell Higgs couplings are the same as those on-shell, ATLAS and CMS data impose upper limits $\Gamma_{H}<(5.4,5.5) \times\left.\Gamma_{H}\right|_{\mathrm{SM}}$. However, certain of the higher-dimensional operators in the SM EFT can modify significantly the distributions for $W W$ and $Z Z$ final states, and a model-independent approach to bounding $\Gamma_{H}$ would require making a global fit including their coefficients [56].

Results from one global fit to SM EFT coefficients including precision electroweak data, the kinematics of associated $H V$ production and LHC TGC measurements as well as Higgs coupling measurements are shown in the left panel of Fig. 8 [57], and results for constraints on TGCs from including Higgs data are shown in the right panel of Fig. 8 [58]. The latter illustrates nicely the complementarity between TGC and Higgs data.

The SM EFT is the most powerful tool to parameterize and search for possible physics beyond the SM in the electroweak and Higgs sectors, and the LHC Higgs Cross-Section Working Group is developing recommendations for how to apply it in a standard way. 

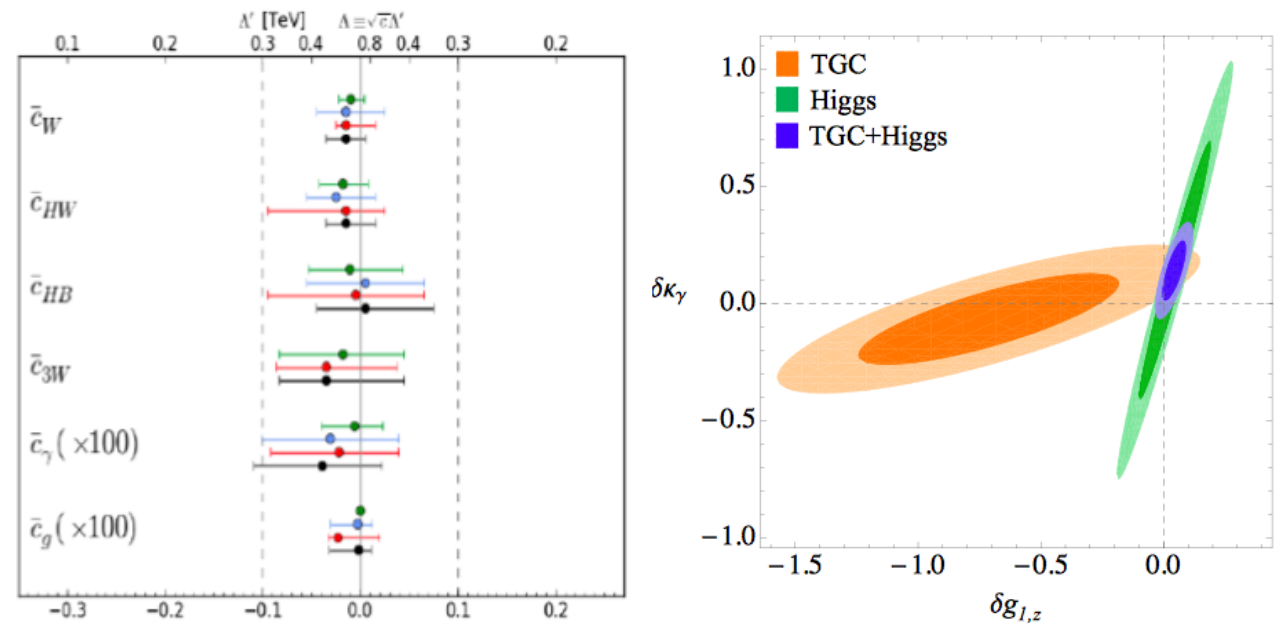

Figure 8: Left panel: Results from a global fit to the coefficients of dimension-6 operators in the SM EFT: the green bars are for each operator individually, and the other bars were obtained including all operators, using Higgs production data (blue), TGCs (red) and all data (black) [57]. Right panel: Comparison of the constraints on anomalous TGCs from TGC data alone (orange) and including Higgs data (blue) [58].

\section{Escaping the Bondage of the SM}

To paraphrase James Bond, "The Standard Model is not enough". There are many concrete physical arguments for this, of which I list (inevitably) just 007. 1) The electroweak vacuum is not stable in the SM, if one take at face value the measurements of $m_{t}$ and $m_{H}$ and extrapolates naively to high scales without introducing new physics. 2) The SM has no candidate for cosmological dark matter. 3) The SM does not explain the origin of matter. 4) The SM does not include the mixing and masses of the neutrinos. 5) The SM does not explain the origin and naturalness of the hierarchy of mass scales in physics. 6) The SM does not accommodate cosmological inflation. 7) The SM does not include a quantum theory of gravity.

In the following, I discuss some of these issues, many of which will be addressed during Run II of the LHC, and most of which would be at least mitigated by supersymmetry, which remains my favourite framework for physics beyond the SM.

\section{The End of the Universe may be Nigh}

The quartic self-coupling $\lambda$ in the SM Higgs potential is renormalized by itself and, more importantly, by its coupling to the top quark. This renormalization is negative, and tends to drive $\lambda<0$ at a scale $\Lambda_{I}$ estimated in [59] to be

$$
\log _{10}\left(\frac{\Lambda_{I}}{\mathrm{GeV}}\right)=11.3+1.0\left(\frac{m_{H}}{\mathrm{GeV}}-126\right)-1.2\left(\frac{m_{t}}{\mathrm{GeV}}-173.10\right)+0.4\left(\frac{\alpha_{s}\left(m_{Z}\right)-0.1184}{0.0007}\right) .
$$

Uisng the central values of the current world averages of $m_{t}(2.1), m_{H}$ (5.1) and $\alpha_{s}$, it seems that the current electroweak vacuum is metastable, as seen in Fig. 9, and adding the uncertainties in 
quadrature in (7.1) (including $\left.\Delta \alpha_{s}\left(m_{Z}\right)= \pm 0.0006\right)$ one estimates

$$
\log _{10}\left(\frac{\Lambda_{I}}{\mathrm{GeV}}\right)=11.1 \pm 1.3
$$

The new CMS measurement of $m_{t}$ [13] would modify this to $\log _{10}\left(\Lambda_{I} / \mathrm{GeV}\right)=11.6 \pm 0.7$ (the uncertainty would increase to \pm 0.9 if the uncertainty in $\alpha_{s}$ were doubled). According to the SM, Higgs field values above this scale have a lower energy than the current SM vacuum, which is therefore unstable. The estimate (7.2) will require re-evaluation when the new CMS, D0 and ATLAS values of $m_{t}$ are incorporated in an updated world average, but should one worry at all about this apparent instability? After all, the lifetime for vacuum decay is probably much longer than the age of the Universe (just as well, otherwise we would not be here). However, I do consider this to be a problem, for two reasons. One is that if the true vacuum is at some large Higgs value, how come our present vacuum energy is very small in natural units? (Please do not throw an anthr*p*c argument at me!) More cogently, if there is a lower-energy state out there, one would have expected (almost all of) the Universe to have fallen into it because of the large quantum or thermal fluctuations in the hot and dense early Universe [60]. (Again, please do not throw an anthr*p*c argument at me!) Averting this disaster requires some new physics beyond the SM, maybe only dimension- 6 operators or quantum gravity effects [61], but supersymmetry would also do just fine [62].

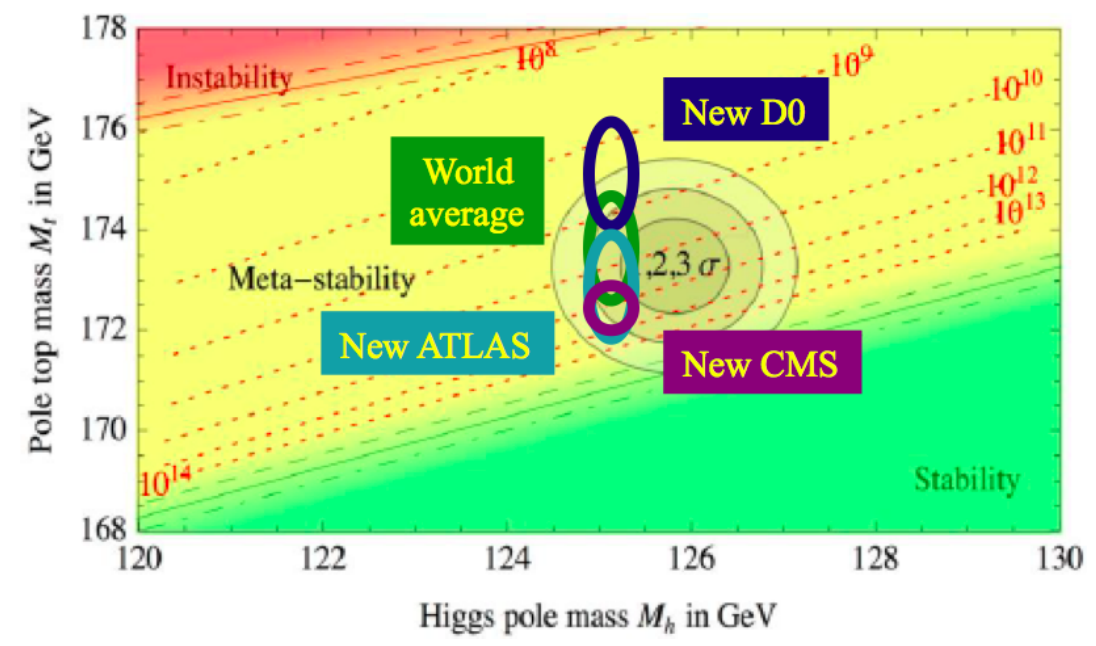

Figure 9: The $\left(m_{H}, m_{t}\right)$ plane showing regions of vacuum (meta/in)stability [59] and the 68\% CL regions favoured by LHC measurements of $m_{H}$ [45] and the 2014 world average as well as more recent measurements of $m_{t}[9]$.

\section{Supersymmetry: Dusk or Dawn?}

Supersymmetry still seems to me the most attractive scenario for physics beyond the SM, and I would argue that Run 1 of the LHC has provided 3 additional motivations: supersymmetry would stabilize our electroweak vacuum [62], simple supersymmetric models predicted correctly that the mass of the Higgs boson would be $\lesssim 130 \mathrm{GeV}$ [63], and they also predicted correctly that its couplings would be very similar to those in the SM [64]. These are new arguments in addition to 
the familiar arguments of naturalness, GUTs, superstrings and dark matter. Of course, we would be on safer ground if supersymmetry had appeared during Run 1, but the conventional missing-energy searches have revealed nothing so far :( Should one be considering exotic signatures that might have been missed, or just push on hopefully to higher masses?

The standard missing-energy signature is weakened in models with compressed spectra, and ATLAS and CMS have been looking intensively at the possibility that the mass difference between the lighter stop squark, $\tilde{t}_{1}$ and the lightest supersymmetric particle (LSP) $\chi_{1}^{0}$ might be small [65], as in 'natural supersymmetry' models [66]. Depending how small, many different $\tilde{t}_{1}$ decays are potentially important, and it is possible that the $\tilde{t}_{1}$ has fallen through the cracks in the searches, as seen in Fig. 10. Interestingly, calculations and measurements of the $\bar{t} t$ spin correlations are now sufficiently precise to exclude one corner of this mosaic [67]. Searches for $\tilde{t}_{1} \rightarrow t+\chi_{1}^{0}$ and $\tilde{t}_{1} \rightarrow b+$ chargino show promise for extending this search during Run II [68].

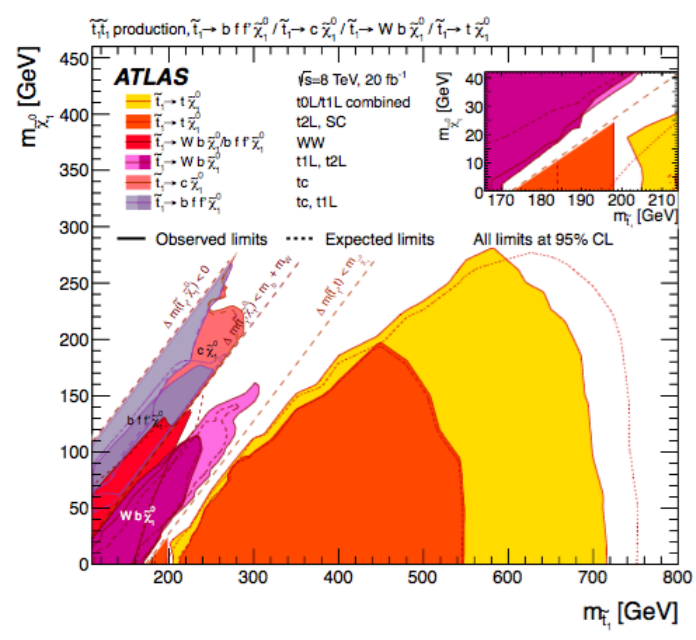

Figure 10: The $\left(m_{\tilde{t}_{1}}, m_{\chi_{1}^{0}}\right)$ plane showing the regions explored in various searches during LHC Run $i$ [65], as well as the triangular region excluded by a measurement of the $\bar{t} t$ spin correlation [67].

Another possibility is that the mass difference between the next-to-lightest sparticle (NLSP) and the LSP may be so small that the LSP may show up in an LHC detector as a long-lived particle with a separated decay vertex, or could even barrel out of the detector as a massive metastable charged particle [65]. Searches for such signatures have often been interpreted in models where the NLSP is a chargino, but there are also scenarios where it could be a charged slepton such as the lighter stau, $\tilde{\tau}_{1}[68]$.

Although these searches have not found anything yet, other supersymmetry-motivated searches have found anomalies to whet the appetite [65]. For example, CMS has reported an 'edge' feature in a dilepton spectrum [69], and ATLAS saw an excess of $Z$ events with missing transverse energy [70]. Let us see what happens at Run II.

The same caution applies to other anomalies that have no reasonable supersymmetric interpretation, such as the excesses of jet combinations with invariant masses around 2 and $5.2 \mathrm{TeV}[71,4$, 25]. 


\section{Energy Frontier vs Intensity Frontier}

There are many examples of light, weakly-interacting particles that are difficult to detect at the LHC, but potentially accessible to high-intensity lower-energy experiments [72] - though important constraints on some of these models are imposed by collider experiments such as BaBar and, most recently, LHCb. Examples include 'dark photons', light dark matter particles, right-handed neutrinos and low-mass, weakly-coupled $Z^{\prime}$ bosons. Some of these models are of particular interest for explaining the discrepancy in $g_{\mu}-2$. Unfortunately, as seen in Fig. 11, at least the dark photon interpretation of $g_{\mu}-2$ seems now to be excluded.

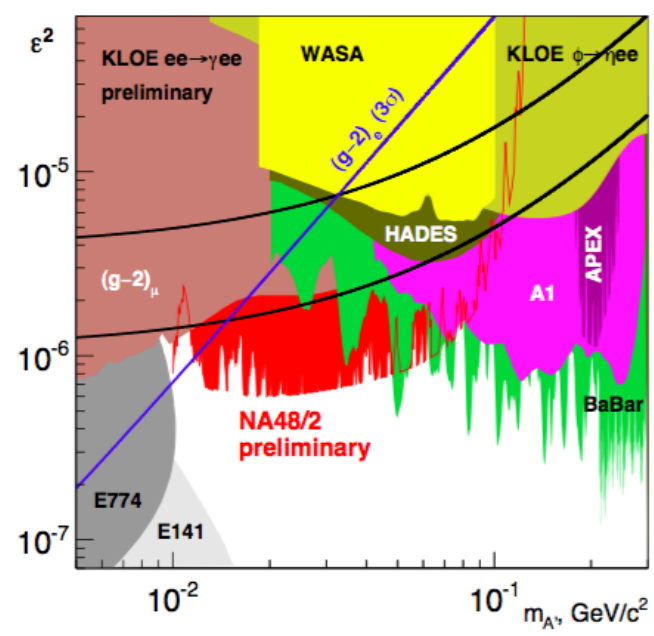

Figure 11: The mass vs mixing plane for dark photon models, exhibiting the many experimental constraints, which seem to exclude the region of interest for interpreting the $g_{\mu}-2$ discrepancy [72].

Projects for fixed-target or beam-dump experiments include HPS, DarkLight and BDX. A particularly ambitious new project is SHiP [73], which would be able to explore many of these scenarios and also have some bread-and-butter $v_{\tau}$ physics. There are also possibilities in the NA62 experiment that is designed to measure $K \rightarrow \pi v \bar{v}$, and ideas for installing a (low-energy) in an underground laboratory and looking in a nearby large neutrino or proton decay detector for light, weakly-interacting particles that it could produce [72].

It is important to pursue such off-the-beaten-track ideas, particularly in view of the growing uncertainty what new physics beyond the SM may be awaiting us at the energy frontier.

\section{Neutrino Physics}

This is the one area away from the energy frontier where new physics beyond the SM [74] has been discovered, in the form of neutrino mixing and (presumably) masses, and experimental progress is being made rapidly.

In particular, great progress has been made with the measurement of $\theta_{13}$ in the Daya Bay [75], RENO [76] and Double Chooz [77] reactor experiments [78]. The promising next step is the JUNO experiment [79], at a distance intermediate between KamLAND and the current experiments, where both low-frequency 'solar' and high-frequency 'atmospheric' neutrino oscillations will be important. 
Progress on high-frequency oscillations was also reported at this Symposium [80], with the observation of a fifth $v_{\mu} \rightarrow v_{\tau}$ event by OPERA [81], amounting to a $5 \sigma$ discovery of this effect, and 'first light' from the NOvA experiment [82]. With a fraction of the planned number of events, NOvA has already obtained a constraint in the $\left(\sin ^{2} \theta_{23}, \Delta m^{2}\right)$ that is comparable with those from MINOS and T2K, as seen in Fig. 12. The combination of T2K with reactor neutrino data already gave a mild preference for the normal hierarchy of neutrino masses and for a non-zero CP-violating phase $\delta \sim 3 \pi / 2[83,80]$, and both these apparent trends are compatible with the NOvA data. We are a long way from a $5 \sigma$ discovery of $\mathrm{CP}$ violation in neutrino oscillations, which will presumably require a new facility such as LBNF or HyperKamiokande [86], but the augurs are promising.

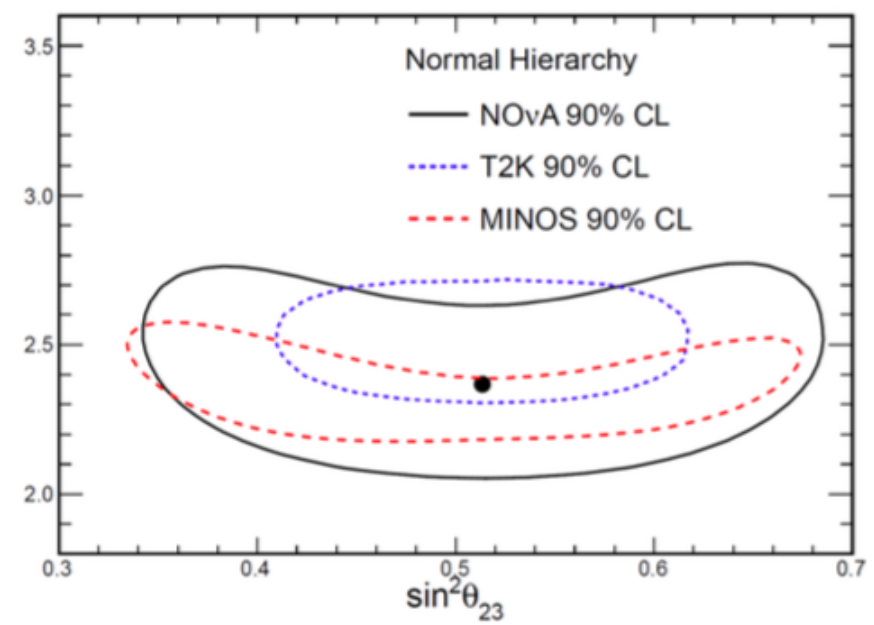

Figure 12: First preliminary data from the NOvA accelerator neutrino experiment [82], compared with previous data from the T2K [83] and MINOS [84] experiments.

Promising progress in the measurement of the absolute neutrino mass scale seems possible in the (relatively) near future [85]. The grandiose Katrin experiment to measure the end-point of the Tritium $\beta$-decay spectrum is scheduled to start taking data in 2016, and should have a 90\% CL upper limit sensitivity $\sim 0.2 \mathrm{eV}$ after a full year of beam time. There are several projects to measure the neutrino mass via $e^{-}$capture on ${ }^{163} \mathrm{Ho}$. In parallel, several experiments are pursuing a possible Majorana neutrino mass via neutrinoless double- $\beta$ decay, and their combination has reached an upper limt $\sim 0.13 \mathrm{eV}$ at the $90 \% \mathrm{CL}$. Extensions of such experiments should eventually be able to reach into the range allowed by models with an inverted hierarchy of neutrino masses, but the range allowed with the normal mass hierarchy would be a challenge [85]. Meanwhile, the Planck measurements of the CMB have been used to set an upper limit on the sum of neutrino masses of $\sim 0.2 \mathrm{eV}$ at the $68 \% \mathrm{CL}$, and a possible future CMB mission, the Cosmic Origins Explorer (COrE) could reach a sensitivity $<60 \mathrm{meV}$ and measure the absolute value of the sum of neutrino masses [87].

\section{Novel Ideas}

In this Section I describe 3 theoretical ideas that are novel: the latter two are very new, but the first has been around for a decade, but has only recently been the object of a surge of interest. 


\subsection{Twin Higgs and Neutral Naturalness}

The naturalness problem arises in the SM because quantum corrections to the squared mass of the Higgs boson are out of control [88]: in particular, there is a net quadratic divergence dominated by the heavy top quark, which is cancelled in a supersymmetric theory by the stop squarks. The twin Higgs idea [89] is to introduce a different symmetry, again by doubling the number of degrees of freedom, but this time with particles that are neutral under the SM gauge group: instead, they form a copy of the SM with isomorphic but distinct couplings. The two copies $A, B$ are then connected via an SU(4) symmetry of the Higgs potential:

$$
V \ni \lambda\left(\left|H_{A}\right|^{2}+\left|H_{B}\right|^{2}\right) .
$$

The idea is then to cancel the SM quadratic divergences with loops of SM-neutral particles: "neutral naturalness". If one then postulates a negative squared mass for $H_{B}$, the underlying SU(4) symmetry is broken down to $\mathrm{SU}(3)$, with 3 true Nambu-Goldstone bosons that are 'eaten' by the $W_{B}^{ \pm}$and $Z_{B}$ and 4 PNGBs that constitute 'our' Higgs doublet. This model serves to broaden one's mind about possible LHC signals, suggesting that the Higgs boson could decay into $B$-sector glueballs with displaced vertices [90].

\subsection{The Relaxion}

This suggestion $[91,92]$ is to add to the SM an axion-like field $\phi$ with quite specific couplings:

$$
\mathscr{L}=\left(M^{2}-g \phi\right)|H|^{2}-g M^{2} \phi+\frac{\phi}{32 \pi^{2} f} \tilde{G} G .
$$

After chiral symmetry breaking, the last term in (11.2) is converted to the quark mass-dependent form

$$
\mathscr{L}_{\chi S B}=f_{\pi}^{3} m_{q} \cos \left(\frac{\phi}{f}\right)=f_{\pi}^{3} \lambda_{q}\langle h\rangle \cos \left(\frac{\phi}{f}\right),
$$

where we have indicated the consequent implicit dependence of $\mathscr{L}_{\chi S B}$ on the Higgs vev. One can now follow the cosmological evolution of the axion-like field $\phi$. It starts by sliding down the linear term in the effective potential until $g\langle\phi\rangle>M^{2}$, at which point the Higgs develops a vev. When this happens, the periodic $\mathscr{L}_{\chi S B}$ term (11.3) switches on and grows with $\langle h\rangle$, trapping both $\phi$ and the Higgs field, as seen in Fig. 13. In particular, the Higgs vev $\langle h\rangle$ is naturally small, without a small parameter having been introduced into the original Lagrangian (11.2). However, this minimal relaxion model has some issues: it requires some $10^{40}$ e-folds of inflation during the evolution, and the ground state has $\theta_{Q C D} \neq 0$. The model therefore needs epicycles, such as a new QCD-like sector [93]. In itself, however, the idea is very cute, and may have applications elsewhere, e.g., in supersymmetric models [94].

\subsection{Composite Gauge Bosons}

It had long been thought that composite gauge bosons were impossible [95, 96], but then along came an explicit supersymmetric example with and SU(4) gauge group and $6(\mathbf{4}+\overline{\mathbf{4}})$ multiplet pairs, which yielded an SU(2) composite gauge theory with 122 fermions and 32 singlet scalars in the infrared limit. This behaviour was initially a puzzle, although consistent with the 4dimensional $c$-theorem. However, it is now known to be just one example of a strong-weak duality 


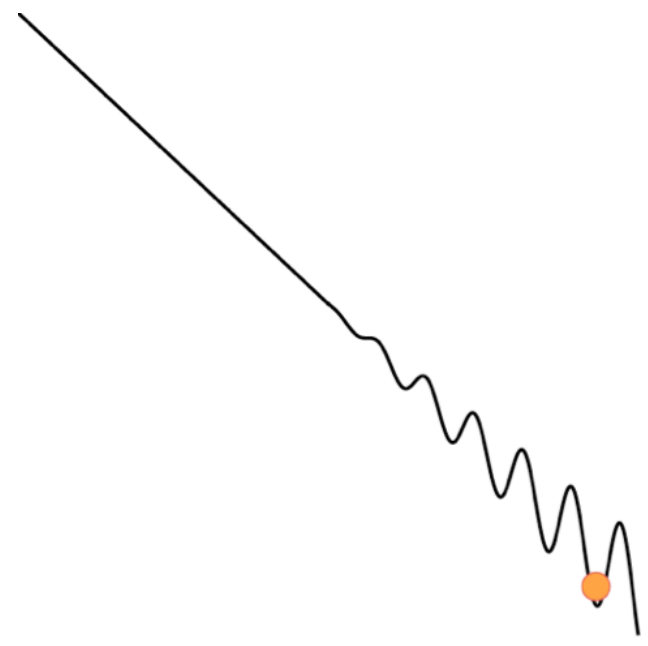

Figure 13: The effective potential for the relaxion $\phi$ [91]. After sliding down the linear part of the potential, the relaxion is trapped in a local minimum once chiral symmetry is broken.

whose proof involves an abstruse relationship between elliptic hypergeometric Gamma functions and q-Pochhammer symbols (!) that the mathematicians have only recently discovered. It has been suggested that some deformation of this construction might be applicable to the $\rho$ meson of QCD [96] - it has long been known that vector meson dominance requires an effective dynamical local symmetry. Or perhaps this construction would be interesting in dynamical models of electroweak symmetry breaking? Might the gauge bosons of the SM actually be composite?

\section{Cosmology}

We have already discussed how CMB measurements can provide important constraints on the neutrino mass scale, and the CMB also constrains the number of neutrino species [87]. In addition, the spectrum of fluctuations in the CMB provides important information on the total density of the Universe (via the multipole moment at which the first peak appears), requiring the existence of dark energy [97], and the densities of ordinary and dark matter (via the relative heights of the other peaks in the spectrum).

These informative CMB fluctuations are thought to be due to an early inflationary epoch [98], and provide a window into possible physics at an energy scale far beyond any conceivable accelerator. The data from the Planck satellite and other experiments on the tilt in the spectrum of scalar perturbations, $n_{s}$ and the tensor-to-scalar ratio $r$ provide important constraints on models of inflation, excluding many simple models based on simple powers of a single inflaton field, as seen in Fig. 14 [99]. However, a number of models survive, notably including the Starobinsky model that is based on a generalization of the Einstein action for General Relativity with an extra $R^{2}$ term [100]. Very similar predictions would be made by models of Higgs inflation [101] if they had a positive potential at large scales. However, as discussed earlier, the effective potential of the SM seems likely to turn negative at some intermediate scale, requiring some epicycle in the original minimal model of Higgs inflation, such as supersymmetry [102]. Inflationary models based on noscale supergravity can also make predictions indistinguishable from those of the $R^{2}$ model [103]. 
Interestingly,as seen in Fig. 14, the experimental limits on $n_{s}$ and $r$ begin to provide constraints on the number of e-folds during inflation, and hence the rate of inflaton decays into lighter particles, which might provide another interesting connection between cosmology and particle physics [104].

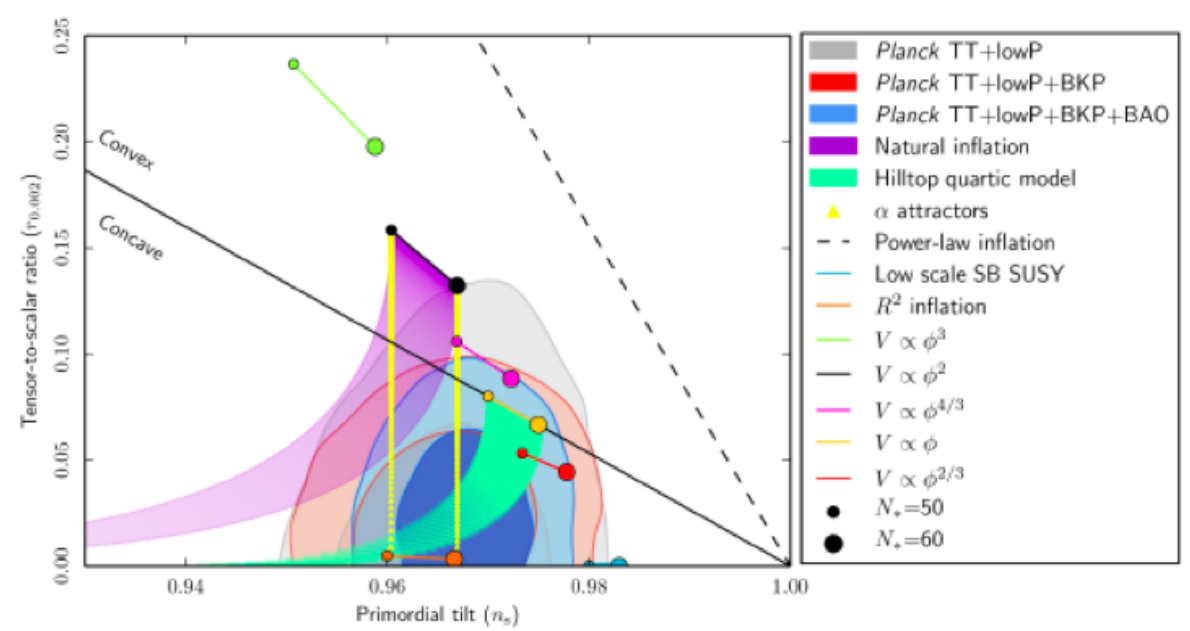

Figure 14: The measurements of the tilt in the scalar CMB perturbations, $n_{s}$, and the tensor-to-scalar ratio, $r$, constrain models of inflation [98]. In particular, simple power-law potentials are disfavoured [99], whereas the Starobinsky $R^{2}$ model [100] is highly consistent with the data, as is Higgs inflation [101] and models based on no-scale supergravity [103].

\section{Gravitational Waves}

Nobody doubts the existence of gravitational waves, particularly since binary pulsars provide strong indirect evidence for them [105]. A non-zero value of $r$ would be evidence for gravitational waves of quantum origin in the CMB, but the BICEP2 hopes have turned to dust [106]. There are several laser spectrometers such as LIGO, Virgo, GEO600 and KAGRA, and advanced LIGO may soon have the capability to detect gravitational waves from mergers of neutron stars and/or black holes. Another way to search for gravitational waves is via pulsar timing, which is closing in on predictions based on black hole mergers, which are also among the prime targets of the future eLISA experiment. We may not have long to wait before gravitational waves are discovered, and the new window of gravitational astronomy opens on the Universe!

\section{Dark Matter}

There are many candidates for the dark matter, whose masses and coupling cover a large range. Here I just focus on the fashionable WIMP scenario,as exemplified by the LSP. If WIMP self-annihilations bring its density into the range allowed by cosmology, the annihilation crosssection should be $\sim 3 \times 10^{-26} \mathrm{~cm}^{2}$ [107]. However, in some models such as supersymmetry, coannihilations with other particles that are almost degenerate could also be important, in which case the self-annihilation cross-section could be smaller.

Fig. 15 compiles the limits from many searches for the spin-independent direct scattering of WIMPs on nuclei [108]. Also shown is the 'neutrino floor', below which there is a background 
from astrophysical neutrinos. The present limits on the scattering cross-section are still some 3 orders of magnitude above this floor, but there are projects with sensitivities approaching it.

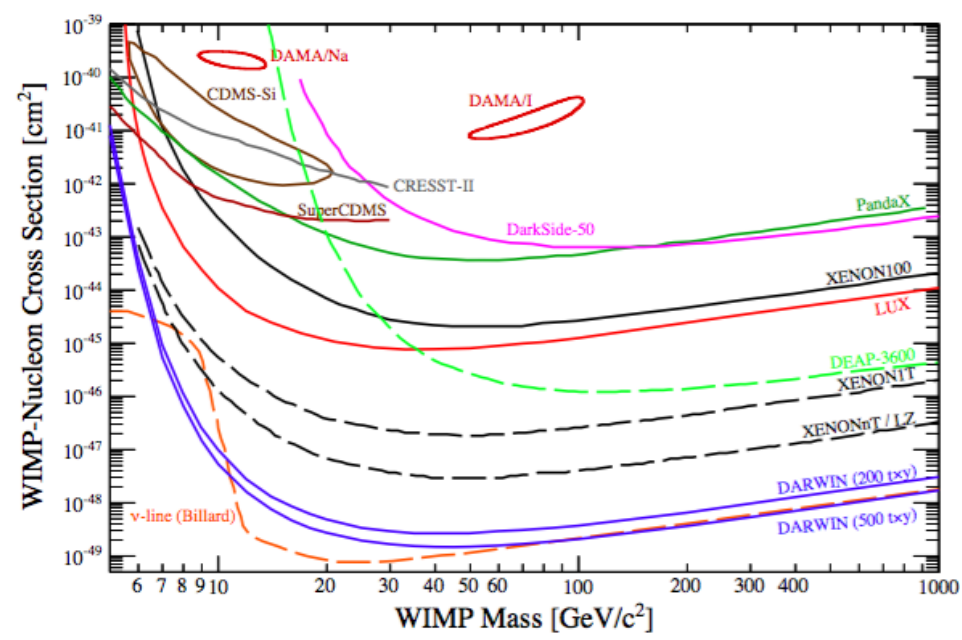

Figure 15: A compilation of present limits and prospective future sensitivities to spin-independent dark matter scattering [108]. The dashed orange line is the 'neutrino floor'.

It is interesting to compare the direct searches for dark matter with those at the LHC using the monojet signature [109]. In the cases of WIMPs that scatter spin-independently, the direct searches are more sensitive unless the WIMP mass $\lesssim 10 \mathrm{GeV}$. However, the LHC monojet searches are much more competitive in the spin-dependent case. Caution is in order, however, since these comparisons are somewhat model-dependent, being sensitive to the mass and couplings of whatever particle mediates the dark matter interaction with conventional matter.
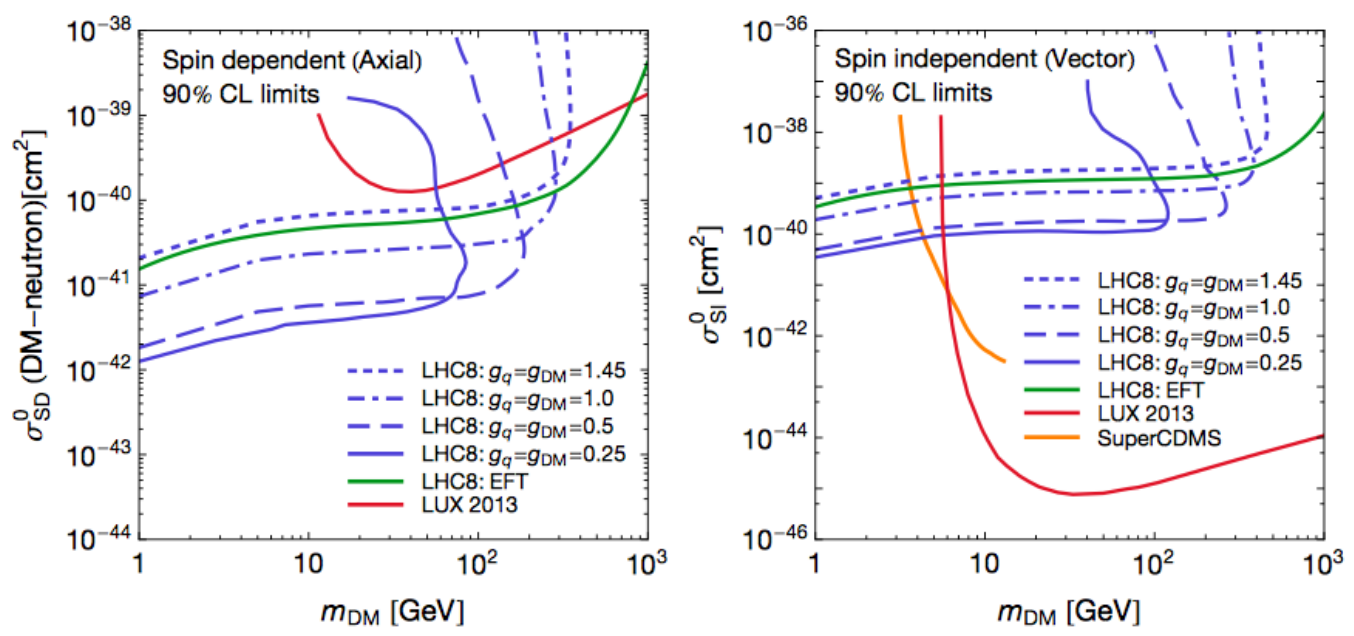

Figure 16: A comparison of the sensitivities to spin-dependent (left panel) and spin-independent (right panel) scattering of dark matter particles with LHC searches for monojets [109].

There have been many claims of indirect signals for the annihilations of astrophysical dark matter particles. For example, there is a well-established excess of $\gamma$-rays from the neighbourhood of the galactic centre in the GeV energy range [110]. However, there is evidence that this excess 
could be due to unresolved point astrophysical sources [112]. The Fermi-LAT Collaboration has also looked for $\gamma$-rays from dark matter annihilations [113], notably by combining data from dwarf galaxies, establishing an upper limit on the cross-section for WIMP annihilations into $\tau^{+} \tau^{-}$that is below $3 \times 10^{-26} \mathrm{~cm}^{2}$ for $m_{\text {WIMP }} \lesssim 70 \mathrm{GeV}$ [110]. There are also interesting limits dark matter self-annihilations from the CMB.

Other options for dark matter searches include looking for positrons and antiprotons produced by annihilations in the galactic halo. The AMS-02 experiment has confirmed previous evidence for a large positron-to-electron ratio in the cosmic rays, but this could be due to a nearby astrophysical source. The same experiment has recently reported a high-statistics measurement of the antiprotonto-proton ratio, which is higher than estimated in previous calculations assuming primary matter cosmic rays [111], but consistent with more recent estimates of the cosmic-ray background [114].

On the other hand, the primary interest in looking for high-energy astrophysical neutrinos now seems to be to search for astrophysical sources [115].

\section{Future LHC Runs}

LHC Run II at $13 \mathrm{TeV}$ is underway. It is worth remembering the enormous amount of work done during the long shutdown, and one must be philosophical about the inevitable frustrations with SEUs in the QPS, TDIs, UFOs, a ULO and earth faults. A total luminosity of $\mathscr{O}(3) / \mathrm{fb}$ was accumulated in 2015, in line with the expectations of [116]. This is exciting because, for some types of heavy new physics weighing over $2 \mathrm{TeV}, 1 / \mathrm{fb}$ at $13 \mathrm{TeV}$ already provides more reach than the 20/fb accumulated at 8/fb in Run I, as seen in Fig. 17.

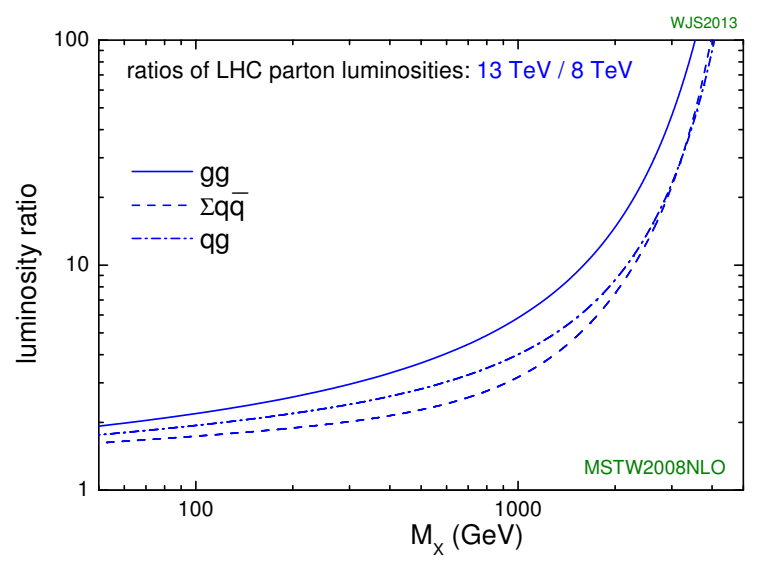

Figure 17: The ratios of various parton-parton luminosities at 13 and $8 \mathrm{TeV}$, as functions of the subprocess invariant masses [4, 25].

ATLAS [25] and CMS [4] have presented their first Run II measurements, including measurements of the total cross-sections for $W^{ \pm}, Z^{0}$ and $\bar{t} t$ production. So far, all these early measurements are consistent with SM predictions. However, each experiment has a showcase dijet event with an invariant mass $\sim 5.2 \mathrm{TeV}$ to titillate the community. 
The LHC is expected to accumulate $\sim 100 / \mathrm{fb}$ in Run I, and reach $300 / \mathrm{fb}$ by the end of Run II, after which the high-luminosity LHC should reach a total of 3000/fb. So the LHC adventure has barely begun, with the energy already increased by a big factor and more than two orders of magnitude of luminosity being planned. In addition to pushing much further the direct searches for new physics beyond the SM, the future LHC runs will provide much more detailed measurements of Higgs properties. Together, these major elements of the future LHC programme will set the stage for possible future collider projects.

\section{Future Accelerators}

The next collider to come online [117] will be the Super KEK-B, which will soon take many flavour measurements to a new level, complementary to LHCb [118].

Looking further ahead, there is general interest in a higher-energy $e^{+} e^{-}$collider. the most mature project is the ILC proposed for Japan [119], aiming initially at a centre-of-mass energy of $500 \mathrm{GeV}$ and eventually $1000 \mathrm{GeV}$. One alternative is the CLIC project being developed by a collaboration including CERN, which is aiming at $3 \mathrm{TeV}$ in the centre of mass [120], but is a less mature project. Recently interest has grown in a possible circular $e^{+} e^{-}$collider, which would be limited to a centre-of-mass energy $\lesssim 350 \mathrm{GeV}$ but could achieve a higher luminosity than the current linear collider designs [121]. One project is the CEPC proposed in China, which would have a circumference of 50 to $70 \mathrm{~km}$ [122]. Another project is the FCC-ee collider being studied as a possible future CERN project [123], which would be located in a tunnel of 80 to $100 \mathrm{~km}$ around Geneva, and aims at an even higher luminosity using technologies being deployed at Super KEK-B.

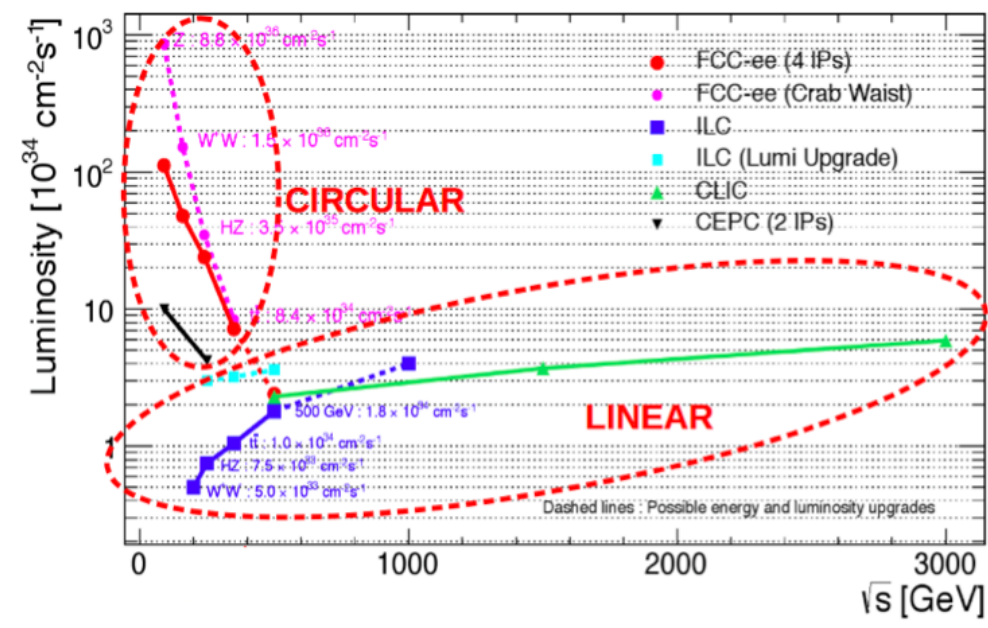

Figure 18: A comparison of the possible centre-of-mass energies of proposed linear and circular $e^{+} e^{-}$ colliders.

Should the worldwide community prioritize energy or greater luminosity at lower energies? Run II of the LHC will provide crucial input. If the LHC discovers some new physics beyond the $\mathrm{SM}$, priority would go to studying it in more detail. If this new physics includes particles light enough to be produced at an $e^{+} e^{-}$collider, that would surely be a top priority. If no such physics 
appears, studying the Higgs, $W^{ \pm}$and $Z^{0}$ in the greatest possible detail would assume a higher priority.

A large circular tunnel would also open up the possibility of a future higher-energy $p p$ collider. For example, 16-Tesla magnets in a 100-km circumference tunnel, as in the FCC-hh project now under study, would enable a centre-of-mass energy of $100 \mathrm{TeV}$ to be reached [121]. With an integrated luminosity target of 20/ab [124], such a collider would provide enormous numbers of Higgs bosons, and would have unequalled reach for new heavy particles. In combination, such a high-energy $p p$ collider and an $e^{+} e^{-}$collider present a compelling vision for the long-term future of high-energy physics, making possible both the direct and indirect exploration of the $10 \mathrm{TeV}$ scale.

Realizing such a vision will require co-operation among the different regions, engaging deciders and the general public, and welcoming new communities and generations [125].

\section{References}

[1] J. F. Grosse-Oetringhaus, talk at this meeting, https://indico.cern. ch/event/325831 /session/9/contribution/24/attachments/1141087/1635020/softQCDp.pdf.

[2] L. Perrone, talk at this meeting, https://indico.cern.ch/event/325831/session/ 15/contribution/47/attachments/1142971/1637735/ Perrone-LeptonPhoton_Perrone.pdf.

[3] V. Khachatryan et al. [CMS Collaboration], arXiv:1507.05915 [hep-ex].

[4] L. Malgeri, talk at this meeting, https://indico.cern.ch/event/325831/session/0/ contribution/9/attachments/1140445/1635019/LP2015_CMSRun2.pdf.

[5] S. de Visscher, talk at this meeting, https://indico.cern.ch/event/325831/session/ 9/contribution/23/attachments/1141150/1634749/LP2015_sdevisscher.pdf.

[6] G. Zanderighi, talk at this meeting, https://indico.cern.ch/event/325831/session/ 9/contribution/25/attachments/1141212/1634762/Zanderighi_LP2015.pdf.

[7] S. Dawson, talk at this meeting, https://indico.cern.ch/event/325831/session/5/ contribution/10/attachments/1140376/1633291/dawson_lp.pdf, arXiv:1511.04040 [hep-ph].

[8] C. Anastasiou, C. Duhr, F. Dulat, F. Herzog and B. Mistlberger, Phys. Rev. Lett. 114 (2015) 212001 [arXiv:1503.06056 [hep-ph]].

[9] ATLAS, CDF, CMS and D0 Collaborations, arXiv:1403.4427 [hep-ex].

[10] K. Melnikov, talk at this meeting, https://indico.cern.ch/event/325831/session/ $10 /$ contribution/30/attachments/1142175/1636384/melnikov_lp2015.pdf.

[11] P. Marquard, A. V. Smirnov, V. A. Smirnov and M. Steinhauser, Phys. Rev. Lett. 114 (2015) 14, 142002 [arXiv:1502.01030 [hep-ph]].

[12] A. Meyer, talk at this meeting, https://indico.cern.ch/event/325831/session/10 /contribution/32/attachments/1142172/1657679/1p2015_topproperties_150820.pdf.

[13] V. Khachatryan et al. [CMS Collaboration], arXiv:1509.04044 [hep-ex]. 
[14] R. Aaij et al. [LHCb Collaboration], Phys. Rev. Lett. 115 (2015) 072001 [arXiv:1507.03414 [hep-ex]]; T Skwarnicki, talk at this meeting, https://indico.cern.ch/event/ 325831 /session/0/contribution/142/attachments/1140432/1634128/ Pentaquarks-LP15-TSkwarnicki_v2.pof.

[15] A. Bondar, talk at this meeting, https://indico.cern.ch/event/325831/session/9/ contribution/26/attachments/1141429/1635109/A.Bondar_LP2015_final.pdf.

[16] M. Karliner and J. L. Rosner, arXiv:1506.06386 [hep-ph].

[17] Qian Wang, Xiao-Hai Liu, Qiang Zhao [arXiv:1508.00339],V.Kubarovsky, M.Voloshin [1508.00888],M.Karliner/J.Rosner [arXiv:1508.01496]

[18] A. Vuorinen, talk at this meeting, https://indico.cern.ch/event/325831/timetable/\#20150820.detailed, then link to Vuorinen_LP2015.pptx, arXiv:1511.01273 [hep-ph].

[19] A. Ukawa, talk at this meeting, https://indico.cern.ch/event/325831/session/9/ contribution/28/attachments/1141210/1634760/Ukawa_LP2015_FTLQCD.pdf.

[20] R. Arnaldi, talk at this meeting, https://indico.cern. ch/event/325831/session/12 /contribution/39/attachments/1142367/1636698/LP2015_Arnaldi.pdf.

[21] P. Kovtun, D. T. Son and A. O. Starinets, Phys. Rev. Lett. 94 (2005) 111601 [hep-th/0405231].

[22] V. Khachatryan et al. [CMS Collaboration], JHEP 1009 (2010) 091 [arXiv:1009.4122 [hep-ex]].

[23] G. Aad et al. [ATLAS Collaboration], arXiv:1509.04776 [hep-ex].

[24] K. Dusling, P. Tribedy and R. Venugopalan, arXiv:1509.04410 [hep-ph].

[25] B. Heinemann, talk at this meeting, https://indico.cern.ch/event/325831/session/ 0/contribution/8/attachments/1140426/1637604/LP2015.pdf.

[26] K. Miyabayashi, talk at this meeting, https://indico.cern.ch/event/325831/ session/11/contribution/34/attachments/1142156/1636864/km_lp2015v7.pdf.

[27] Z. Ligeti, talk at this meeting, https://indico.cern.ch/event/325831/session/11/ contribution/33/attachments/1142267/1636537/LIGETI-LPtalk.pdf.

[28] M. Czakon, P. Fiedler and A. Mitov, Phys. Rev. Lett. 115 (2015) 5, 052001 [arXiv:1411.3007 [hep-ph]].

[29] C. DeTar, talk at this meeting, https://indico.cern.ch/event/325831/session/9/ contribution/27/attachments/1141636/1635489/DETAR-LP2015.pdf.

[30] G. Lanfranchi, talk at this meeting, https://indico.cern.ch/event/325831/session/ 11/contribution/35/attachments/1142177/1636387/LP2015_Lanfranchi.pdf.

[31] V. Khachatryan et al. [CMS and LHCb Collaborations], Nature 522 (2015) 68 [arXiv:1411.4413 [hep-ex]].

[32] V. Khachatryan et al. [CMS Collaboration], Phys. Lett. B 749 (2015) 337 [arXiv:1502.07400 [hep-ex]].

[33] See, for example, A. Paul, https://indico.cern.ch/event/356420/session/3/ contribution/648/attachments/1130541/1615743/BKst11_EPS_2015.pdf, M.Ciuchini, M.Fedele, E.Franco, S.Mishima, A.Paul, L.Silvestrini and M. Valli, to appear. 
[34] For a differing point of view, see S. Descotes-Genon, L. Hofer, J. Matias and J. Virto, arXiv:1510.04239 [hep-ph].

[35] A. J. Buras, M. Gorbahn, S. JŁger and M. Jamin, arXiv:1507.06345 [hep-ph].

[36] R. Tschirhart, talk at this meeting, https://indico.cern.ch/event/325831/session/ $11 /$ contribution/37/attachments/1141621/1636335/ Tschirhart_Lepton-Photon_August_2015_Plenary_final.pdf.

[37] J. Baron et al. [ACME Collaboration], Science 343 (2014) 269 [arXiv:1310.7534 [physics.atom-ph]].

[38] J. Ellis, J. S. Lee and A. Pilaftsis, JHEP 1010 (2010) 049 [arXiv:1006.3087 [hep-ph]]; A. Arbey, J. Ellis, R. M. Godbole and F. Mahmoudi, Eur. Phys. J. C 75 (2015) 2, 85 [arXiv:1410.4824 [hep-ph]].

[39] J. Miller, talk at this meeting, https://indico.cern.ch/event/325831/session/11/ contribution/36/attachments/1142457/1636869/ Miller-Slovenia-Lepton-Flavor-Violation-Experiment-va.pdf.

[40] Fermilab Muon $g-2$ Experiment: http://muon-g-2 f fnal .gov.

[41] For a recent re-evaluation of electron scattering data, see K. Griffioen, C. Carlson and S. Maddox, arXiv:1509.06676 [nucl-ex].

[42] ALEPH, CDF, D0, DELPHI, L3, OPAL, SLD Collaborations, the LEP Electroweak Working Group, the Tevatron Electroweak Working Group and the SLD electroweak and heavy flavour groups, arXiv:1012.2367 [hep-ex].

[43] K. Einsweiler, talk at this meeting, https://indico.cern.ch/event/325831/session/ 7/contribution/17/attachments/1140976/1634311/ LP2015_Electroweak_Summary_LHC_Aug15.pdf.

[44] R. Aaij et al. [LHCb Collaboration], arXiv:1509.07645 [hep-ex].

[45] G. Aad et al. [ATLAS and CMS Collaborations], Phys. Rev. Lett. 114 (2015) 191803 [arXiv:1503.07589 [hep-ex]].

[46] S. Farrington, talk at this meeting, https://indico.cern.ch/event/325831/ contribution/11/attachments/1140999/1634353/sinead_leptonphoton_1608151.pdf.

[47] ATLAS and CMS Collaborations, ATLAS-CONF-2015-044, CMS-PAS-HIG-15-002.

[48] T. Aaltonen et al. [CDF and D0 Collaborations], Phys. Rev. Lett. 114 (2015) 15, 151802 [arXiv:1502.00967 [hep-ex]].

[49] G. Blankenburg, J. Ellis and G. Isidori, Phys. Lett. B 712 (2012) 386 [arXiv:1202.5704 [hep-ph]].

[50] R. Harnik, J. Kopp and J. Zupan, JHEP 1303 (2013) 026 [arXiv:1209.1397 [hep-ph]].

[51] G. Aad et al. [ATLAS Collaboration], arXiv:1508.03372 [hep-ex].

[52] CMS Collaboration, CMS-PAS-HIG-14-040.

[53] Higgs Cross-Section Working Group, https://twiki.cern.ch/twiki/bin/view/ LHCPhysics/Crosssections.

[54] M. Ciuchini, talk at this meeting, https://indico.cern.ch/event/325831/session/ 7/contribution/18/attachments/1140986/1634326/Ciuchini-LP15.pdf.

[55] M. Vidal, talk at this meeting, https://indico.cern.ch/event/325831/session/5 /contribution/12/attachments/1140447/1633440/vidal_lp2015.pdf. 
[56] A. Azatov, C. Grojean, A. Paul and E. Salvioni, Zh. Eksp. Teor. Fiz. 147 (2015) 410 [J. Exp. Theor. Phys. 120 (2015) 354] [arXiv:1406.6338 [hep-ph]].

[57] J. Ellis, V. Sanz and T. You, JHEP 1503 (2015) 157 [arXiv:1410.7703 [hep-ph]].

[58] A. Falkowski, M. Gonzalez-Alonso, A. Greljo and D. Marzocca, arXiv:1508.00581 [hep-ph].

[59] D. Buttazzo, G. Degrassi, P. P. Giardino, G. F. Giudice, F. Sala, A. Salvio and A. Strumia, JHEP 1312 (2013) 089 [arXiv:1307.3536 [hep-ph]].

[60] See, e.g., M. Fairbairn and R. Hogan, Phys. Rev. Lett. 112 (2014) 201801 [arXiv:1403.6786 [hep-ph]]; A. Hook, J. Kearney, B. Shakya and K. M. Zurek, JHEP 1501 (2015) 061 [arXiv:1404.5953 [hep-ph]].

[61] See, e.g., V. Branchina, E. Messina and M. Sher, Phys. Rev. D 91 (2015) 013003 [arXiv:1408.5302 [hep-ph]]. However, see also L. Di Luzio, G. Isidori and G. Ridolfi, arXiv:1509.05028 [hep-ph].

[62] J. R. Ellis and D. Ross, Phys. Lett. B 506 (2001) 331 [hep-ph/0012067].

[63] J. R. Ellis, G. Ridolfi and F. Zwirner, Phys. Lett. B 257 (1991) 83; H. E. Haber and R. Hempfling, Phys. Rev. Lett. 66 (1991) 1815; Y. Okada, M. Yamaguchi and T. Yanagida, Prog. Theor. Phys. 85 (1991) 1.

[64] J. R. Ellis, S. Heinemeyer, K. A. Olive and G. Weiglein, JHEP 0301 (2003) 006 [hep-ph/0211206].

[65] L. Shchutska, talk at this meeting, https://indico.cern. ch/event/325831/session/6 /contribution/14/attachments/1140502/1633555/lp_susy.pdf.

[66] M. Papucci, J. T. Ruderman and A. Weiler, JHEP 1209 (2012) 035 [arXiv:1110.6926 [hep-ph]].

[67] M. Christinziani, talk at this meeting, https://indico.cern.ch/event/325831/ session/10/contribution/31/attachments/1141496/1636623/MC-150820-LP.pdf.

[68] E. A. Bagnaschi et al., arXiv:1508.01173 [hep-ph].

[69] V. Khachatryan et al. [CMS Collaboration], JHEP 1504 (2015) 124 [arXiv:1502.06031 [hep-ex]].

[70] G. Aad et al. [ATLAS Collaboration], Eur. Phys. J. C 75 (2015) 7, 318 [arXiv:1503.03290 [hep-ex]].

[71] B. Kersevan, https://indico.cern.ch/event/325831/session/6/ contribution/15/attachments/1140429/1633410/kersevan-1p2015.pdf.

[72] M. Pospelov, https://indico.cern.ch/event/325831/session/6/ contribution/16/attachments/1140747/1633923/POSPELOV-LeptonPhoton2015.pdf.

[73] S. Alekhin et al. [SHiP Collaboration], arXiv:1504.04855 [hep-ph].

[74] S. Zhou, talk at this meeting, https://indico.cern.ch/event/325831/session/8/ contribution/22/attachments/1141099/1634566/Neutrinos-LP15-ZHOU.pdf.

[75] F. P. An et al. [Daya Bay Collaboration], Phys. Rev. Lett. 108 (2012) 171803 [arXiv:1203.1669 [hep-ex]].

[76] J. K. Ahn et al. [RENO Collaboration], Phys. Rev. Lett. 108 (2012) 191802 [arXiv:1204.0626 [hep-ex]].

[77] Y. Abe et al. [Double Chooz Collaboration], JHEP 1410 (2014) 086 [JHEP 1502 (2015) 074] [arXiv:1406.7763 [hep-ex]].

[78] A. Stahl, talk at this meeting, https://indico.cern.ch/event/325831/session/8/ contribution/19/attachments/1141093/1634550/Stahl-ReactorNeutrinos-V2.pdf. 
[79] Z. Djurcic et al. [JUNO Collaboration], arXiv:1508.07166 [physics.ins-det].

[80] P. Shanahan, talk at this meeting, https://indico.cern.ch/event/325831/session/ 8/contribution/20/attachments/1141064/1634957/Neutrinos ShanahanLP2015.pdf.

[81] N. Agafonova et al. [OPERA Collaboration], arXiv:1507.01417 [hep-ex].

[82] NOvA Collaboration, http://www-nova.fnal.gov.

[83] K. Abe et al. [T2K Collaboration], Phys. Rev. Lett. 112 (2014) 061802 [arXiv:1311.4750 [hep-ex]].

[84] P. Adamson et al. [MINOS Collaboration], Phys. Rev. Lett. 110 (2013) 25, 251801 [arXiv:1304.6335 [hep-ex]].

[85] S. Zavatarelli, talk at this meeting, https://indico.cern.ch/event/325831/session/ 8/contribution/21/attachments/1141098/1634558/zavatarelli_LP2015.pdf.

[86] K. Long, talk at this meeting, https://indico.cern.ch/event/325831/session/18/ contribution/62/attachments/1143175/1638148/LP15-Long.pdf.

[87] J. Delabrouille, talk at this conference, https://indico.cern.ch/event/325831/ session/14/contribution/44/attachments/1142823/1637478/ DELABROUILLE_Measuring-the-CMB.pdf.

[88] M. McCullough, talk at this meeting, https://indico.cern.ch/event/325831/ session/6/contribution/13/attachments/1140468/1633470/LeptonPhoton.pdf.

[89] Z. Chacko, H. S. Goh and R. Harnik, Phys. Rev. Lett. 96 (2006) 231802 [hep-ph/0506256].

[90] N. Craig, A. Katz, M. Strassler and R. Sundrum, JHEP 1507 (2015) 105 [arXiv:1501.05310 [hep-ph]]; P. Schwaller, D. Stolarski and A. Weiler, JHEP 1505 (2015) 059 [arXiv:1502.05409 [hep-ph]].

[91] P. W. Graham, D. E. Kaplan and S. Rajendran, arXiv:1504.07551 [hep-ph].

[92] For kindred earlier ideas, see G. Dvali and A. Vilenkin, Phys. Rev. D 70 (2004) 063501 [hep-th/0304043] and G. Dvali, Phys. Rev. D 74 (2006) 025018 [hep-th/0410286].

[93] ibitemEspinosa:2015eda J. R. Espinosa, C. Grojean, G. Panico, A. Pomarol, O. PujolĹs and G. Servant, arXiv:1506.09217 [hep-ph].

[94] B. Batell, G. F. Giudice and M. McCullough, arXiv:1509.00834 [hep-ph].

[95] S. Weinberg and E. Witten, Phys. Lett. B 96 (1980) 59.

[96] Z. Komargodski, talk at this meeting, https://indico.cern. ch/event/325831/ contribution/29/attachments/1140995/1634343/KOMARGODSKI-Ljubl jana.pdf.

[97] J. Estrada, talk at this meeting, https://indico.cern.ch/event/325831/session/ 14 /contribution/45/attachments/1142767/1637380/je_darkenergy_lp2015.pdf.

[98] A. Guth, talk at this conference, https://indico.cern.ch/event/325831/session/ $14 /$ contribution/43/attachments/1142783/1637413/guth-lp15-fri.pdf.

[99] P. A. R. Ade et al. [Planck Collaboration], arXiv:1502.02114 [astro-ph.CO].

[100] A. A. Starobinsky, Phys. Lett. B 91, 99 (1980).

[101] F. L. Bezrukov and M. Shaposhnikov, Phys. Lett. B 659 (2008) 703 [arXiv:0710.3755 [hep-th]].

[102] J. Ellis, H. J. He and Z. Z. Xianyu, Phys. Rev. D 91 (2015) 2, 021302 [arXiv:1411.5537 [hep-ph]]. 
[103] J. Ellis, D. V. Nanopoulos and K. A. Olive, Phys. Rev. Lett. 111 (2013) 111301 [Phys. Rev. Lett. 111 (2013) 12, 129902] [arXiv:1305.1247 [hep-th]].

[104] J. Ellis, M. A. G. Garcia, D. V. Nanopoulos and K. A. Olive, JCAP 1507 (2015) 07, 050 [arXiv:1505.06986 [hep-ph]].

[105] E. Barausse, talk at this meeting, https://indico.cern.ch/event/325831/ contribution/50/attachments/1140785/1633989/Barausse-lepton_photon.pdf.

[106] P. A. R. Ade et al. [BICEP2 and Planck Collaborations], Phys. Rev. Lett. 114 (2015) 101301 [arXiv:1502.00612 [astro-ph.CO]].

[107] T. Volansky, talk at this meeting, https://indico.cern.ch/event/325831/session/ 13/contribution/40/attachments/1142279/1636557/Volansky-LP. DMTheory.2015.3 pdf.

[108] L. Baudis, talk at this meeting, https://indico.cern.ch/event/325831/session/ 13/contribution/41/attachments/1142364/1636694/baudis_leptonph15.pdf.

[109] O. Buchmueller, M. J. Dolan, S. A. Malik and C. McCabe, JHEP 1501 (2015) 037 [arXiv:1407.8257 [hep-ph]]; S. Malik et al., arXiv:1409.4075 [hep-ex].

[110] D. Berge, talk at this meeting, https://indico.cern.ch/event/325831/session/ 15/contribution/48/attachments/1142852/1637624/Berge. LP15.pdf.

[111] Z. Li, talk at this meeting, https://indico.cern.ch/event/325831/session/15/ contribution/46/attachments/1142813/1637457/lepton-photon_2015_zuhaoli.pdf.

[112] S. K. Lee, M. Lisanti, B. R. Safdi, T. R. Slatyer and W. Xue, arXiv:1506.05124 [astro-ph.HE].

[113] M. Sánchez-Conde, talk at this meeting, https://indico.cern.ch/event/325831/ session/13/contribution/42/attachments/1142459/1636872/ SANCHEZ_CONDE_IndirectDMsearches_LeptonPhoton15_200815_final.pdf.

[114] G. Giesen, M. Boudaud, Y. Genolini, V. Poulin, M. Cirelli, P. Salati and P. D. Serpico, JCAP 1509 (2015) 09, 023 [arXiv:1504.04276 [astro-ph.HE]].

[115] N. van Eijndhoven, talk at this meeting, https://indico.cern. ch/event/325831/ session/15/contribution/49/attachments/1141798/1635765/nve-1p2015.pdf.

[116] M. Lamont, talk at this meeting, https://indico.cern.ch/event/325831/session/0 / contribution/7/attachments/1140418/1633374/LHC-lepton-photon-final.pdf.

[117] M. Demarteau, talk at this meeting, https://indico.cern.ch/event/325831/ session/18/contribution/61/attachments/1143176/1638149/ demarteau_lepton-photon.pdf.

[118] Y. Sakai, talk at this meeting, https://indico.cern.ch/event/325831/timetable/ \#20150820. detailed, then link to lp15_sakai.pptx.

[119] S. Komamiya, talk at this meeting, https://indico.cern.ch/event/325831/session/ 17 /contribution/57/attachments/1142965/1637728/Komamiya_ILC_Ljubljana.pdf.

[120] P. Roloff, talk at this meeting, https://indico.cern.ch/event/325831/session/18/ contribution/59/attachments/1143167/1638133/lepton_photon_2015_roloff.pdf.

[121] M. Benedikt, talk at this meeting, https://indico.cern.ch/event/325831/session/ $18 /$ contribution/60/attachments/1143145/1638099/ 150822-MBE_FutureCircularColliders_ap.pdf. 
[122] CEPC pre-CDR, http://cepc.ihep.ac.cn/preCDR/volume.html.

[123] FCC-ee Design Study, http://tlep.web.cern.ch.

[124] I. Hinchliffe, A. Kotwal, M. L. Mangano, C. Quigg and L. T. Wang, Int. J. Mod. Phys. A 30 (2015) 1544002 [arXiv:1504.06108 [hep-ph]].

[125] K. Shaw, talk at this meeting, https://indico.cern.ch/event/325831/timetable/ \#20150820.detailed, then link to Education_Outreach_IPPOG.pptx. 